\title{
Signatures of magnetostriction and spin-phonon coupling in magnetoelectric hexagonal 15R-BaMnO 3
}

\author{
Bommareddy Poojitha, Anjali Rathore, Ankit Kumar, and Surajit Saha* \\ Department of Physics, Indian Institute of Science Education and Research, Bhopal 462066, India \\ *Correspondence: surajit@iiserb.ac.in
}

\begin{abstract}
Spin-phonon coupling, the interaction of spins with surrounding lattice is a key parameter to understand the underlying physics of multiferroics and engineer their magnetization dynamics. Elementary excitations in multiferroic materials are strongly influenced by spin-phonon interaction, making Raman spectroscopy a unique tool to probe these coupling(s). Recently, it has been suggested that the dielectric and magnetic properties of 15R-type hexagonal $\mathrm{BaMnO}_{3}$ are correlated through the spin-lattice coupling. Here, we report the observation of an extensive renormalization of the Raman spectrum of $15 \mathrm{R}-\mathrm{BaMnO}_{3}$ at $230 \mathrm{~K}, 280 \mathrm{~K}$, and 330 $\mathrm{K}$. Magnetic measurements reveal the presence of a long-range and a short-range magnetic ordering in $15 \mathrm{R}-\mathrm{BaMnO}_{3}$ at $230 \mathrm{~K}$ and $330 \mathrm{~K}$, respectively. The Raman spectrum shows the appearance of new Raman modes in the magnetically ordered phases. Furthermore, an additional Raman phonon appears below $280 \mathrm{~K}$, possibly arising from a local latticedistortion due to the displacement of Mn-ions, that exhibits anomalous shift with temperature. The origin of the observed renormalization and phonon anomalies in Raman spectra are discussed based on the evidences from temperature- and magnetic-field-dependent Raman spectra, temperature-dependent $\mathrm{x}$-ray diffraction, magnetization, and specific heat measurements. Our results indicate the presence of magnetostriction and spin-phonon coupling in $15 \mathrm{R}-\mathrm{BaMnO}_{3}$ thus suggesting that the optical phonons are strongly correlated to its magnetoelectric properties.
\end{abstract}

\section{Introduction}

The coupling among various degrees of freedom such as spin, charge, lattice, and orbital is important as it gives rise to various novel phenomena and leads to exotic ground states in condensed matter systems [1-4]. The interplay between phonons and electron spins is relevant for many exotic phenomena including spin-peierls transition, phonon Hall effect, colossal magnetoresistance, ultrafast magnetization control, and spin Seebeck effect etc. [1-5] Recently, there has been a renewed interest in studying spin-phonon coupling (SPC) within the context of multiferroics and spintronics. In multiferroic materials, SPC has been implored to explain the compelling phenomena such as the thermal Hall-effect [6]. Moreover, SPC allows tailoring the functionalities of transition metal oxides such as stabilizing new multiferroic ground state by applying strain, tuning to unusual magnetic ground states etc. SPC also provides useful information about spin relaxation time which is an important concern in spintronics applications such as quantum computing [7-13]. Unfortunately, the number of candidate (multiferroic) materials is limited and it often happens that either the transitions are at very low temperatures or the response of cross-coupling between ferroic orders is very weak to be useful 
in device applications [14]. Hence, the search for new multiferroic materials with better functionalities is constantly increasing over the period of time.

In this context, the $15 \mathrm{R}$-type hexagonal $\mathrm{BaMnO}_{3}\left(15 \mathrm{R}-\mathrm{BaMnO}_{3}\right)$ has received a renewed importance after the discovery of magnetoelectric property near room temperature [15], exhibiting antiferromagnetism below $\left(\mathrm{T}_{\mathrm{N}}\right) \sim 230 \mathrm{~K}$. It is considered to be an exceptional class of magnetoelectrics due to the fact that both the electric and magnetic properties are associated with the same $\mathrm{Mn}^{+4}$ ions. The electric polarization arises from the displacement of the same ions (magnetic ions $-\mathrm{Mn}^{4+}$ ) along the $c$-axis, akin to that of classic ferroelectric material $\mathrm{BaTiO}_{3}$. It was believed that the asymmetric environment of $\mathrm{Mn}$ ions within $\mathrm{MnO}_{6}$ Octahedra in the cubic and hexagonal layers leads to ferroelectric instability in the lattice which also has an intimate connection with magnetic ordering [15]. Korneta et al. observed that both magnetization and dielectric constant are highly anisotropic and the in-plane (ab-plane) dielectric constant is sensitive to an applied magnetic field [15]. They have attributed the observed correlation between magnetic and dielectric responses to the possible spin-phonon coupling in $15 \mathrm{R}-\mathrm{BaMnO}_{3}$. Since phonons play a major role in ferroelectric materials [16-18], one may expect them to have an equally important role in the magnetoelectric multiferroics too. A detailed understanding of the phonons and their coupling is, therefore, extremely important as it extends the opportunity to engineer new functionalities in transition metal oxides such as $\mathrm{BaMnO}_{3}$ and others.

The idiosyncratic feature of spin-phonon coupling is the renormalization of phonon parameters. The electron spin can interact with its surrounding lattice in a number of possible ways, for instance, the lattice vibrations involving $\mathrm{Mn}-\mathrm{O}$ or $\mathrm{Mn}-\mathrm{O}-\mathrm{Mn}$ bonds modify spin-spin correlations through superexchange interactions in antiferromagnets [19-21]. Similarly, if spins are coupled to elastic degrees of freedom, spontaneous strain occurs at magnetic transition temperature, giving rise to magnetostriction [22]. Multiferroic properties are associated with the lowering of symmetries (spatial and time-reversal symmetry). Since Raman spectroscopy is sensitive to these changes, it emerges as a powerful tool to detect the presence of coupling between different degrees of freedom, e.g., magnetostriction, spin-phonon coupling, and electron-phonon coupling etc. in strongly correlated multifunctional materials [11, 12, 22, 23]. In this article, we have investigated the correlation between the phonons and magnetoelectric properties of $15 \mathrm{R}-\mathrm{BaMnO}_{3}$. Temperature-dependent magnetization measurements reveal two magnetic transitions: a possible short-range ordering at $\mathrm{T}_{\mathrm{S}} \sim 330( \pm 0.2) \mathrm{K}$, hitherto unknown, and a long-range antiferromagnetic ordering at $\mathrm{T}_{\mathrm{N}} \sim 230( \pm 0.2) \mathrm{K}$. We have observed six Raman active modes responding to the magnetic transitions at $\mathrm{T}_{\mathrm{S}}$ (short-ranged ordering) and $\mathrm{T}_{\mathrm{N}}$ (antiferromagnetic ordering) which can be attributed to spin-phonon coupling and magnetostriction. Additionally, a new phonon mode emerges in the Raman spectra at temperatures below $\mathrm{T}_{\mathrm{D}} \sim 280 \mathrm{~K}$, indicating a local lattice distortion possibly arising from displacements of the Mn-ions, the frequency which exhibits anomalous softening with decreasing temperature. Magnetic-field dependence of the Raman modes and temperaturedependent x-ray diffraction measurements corroborate our observations. The strength of spinphonon coupling is estimated by a model based on mean-field and two-spin cluster 
approximations. The origin of a strong renormalization of the Raman active phonons below the magnetic transitions $\left(\mathrm{T}_{\mathrm{S}} / \mathrm{T}_{\mathrm{N}}\right)$ is discussed in detail.

\section{Experimental details}

Polycrystalline samples of $15 \mathrm{R}-\mathrm{BaMnO}_{3}$ were synthesised by using solid-state reaction method. High purity $\mathrm{BaCO}_{3}(99.999 \%)$ and $\mathrm{MnO}_{2}$ (99.99\%) (Sigma-Aldrich) powders were used as precursors. The stoichiometric mixture was ground well for 3 hours and calcined at $1250{ }^{\circ} \mathrm{C}$ and $1300{ }^{\circ} \mathrm{C}$ for 2 hours each with intermediate grindings. After the calcination, the resultant powder was ground, pelletized, and sintered at $1400{ }^{\circ} \mathrm{C}$ for 6 hours. Powder X-ray diffraction (PXRD) measurements were done by PANalytical Empyrean x-ray diffractometer with $\mathrm{Cu}-\mathrm{K}_{\alpha}$ radiation of wavelength $1.5406 \AA$ A. Liquid nitrogen-based Anton Paar TTK 450 heating stage was used to control the sample-temperature during non-ambient PXRD measurements. Chemical compositions were determined using energy dispersive $\mathrm{X}$-ray (EDAX) technique equipped with high-resolution field emission scanning electron microscope (HR-FESEM) (Zeiss ULTRA Plus). Transmission electron microscopy (TEM) images were collected to verify the periodicity in the lattice using TALOS S-FEG (Schottky FEG (Field Emission Gun-Transmission Electron Microscope)) emitter with accelerating voltage of 200 $\mathrm{kV}$. For the TEM measurement of the samples: a small amount of the powder sample of as synthesized compound was dispersed in distilled water by ultrasonicating for $20 \mathrm{~s}$. This (turbid) solution was then drop-casted on a carbon coated copper grid forming a very thin layer. The solution/layer was then allowed to dry for a few hours thoroughly before it was attached to the sample holder on the microscope (TEM) for viewing. Raman spectra were collected from pelletized samples in the backscattering configuration using a LabRAM HR Evolution Raman spectrometer attached with a frequency-doubled Nd:YAG (neodymium-doped yttrium aluminium garnet; $\mathrm{Nd}: \mathrm{Y}_{3} \mathrm{Al}_{15} \mathrm{O}_{12}$ ) laser excitation source of wavelength $532 \mathrm{~nm}$ and Peltier cooled charge-coupled device (CCD) detector. A microscope objective of 50X magnification and numerical aperture of 0.5 was used to collect the scattered light. A Linkam stage (Model HFS600E-PB4) was used for variable temperature Raman measurements. The Raman spectrometer was also optically coupled to a closed cycle cryomagnet (Attocube 1000) to measure magnetic field-dependent Raman spectra at low temperature. DC magnetization measurements as a function of temperature and external magnetic field were carried out using Quantum Design SQUID-VSM (Superconducting Quantum Interference Device with Vibrating Sample Magnetometer). Specific heat $\left(C_{\mathrm{p}}\right)$ measurements were performed using a commercial Quantum Design PPMS on thin, flat pellet samples in the temperature range (T: 5 to $390 \mathrm{~K}$ ). Data were collected in two different runs: the data from 5 to $295 \mathrm{~K}$ were collected in the first run where Apiezon $\mathrm{N}$-grease was used (to stick the sample to the holder) as an addenda and the high temperature data (T: 295 to $390 \mathrm{~K}$ ) were collected in the second run where it needs additional oven attachment and Apiezon H-grease (glue to stick the sample to holder) as an addenda. The data were collected during the heating cycle. Measurement on the sample was preceded by a measurement of the addenda in the same temperature range following a thermal relaxation method. Heat capacity $\left(C_{\mathrm{p}}\right)$ of the sample was obtained after subtracting the addenda heat capacity from total heat capacity. 


\section{Results and Discussion}

\section{Structural and magnetic properties:}

The crystal structure of $15 \mathrm{R}-\mathrm{BaMnO}_{3}$, drawn using VESTA (Visualization of Electronic and STructural Analysis) software [24], is shown in Figure 1(a). It contains 15 formula units (75 atoms) per unit cell and 5 formula units ( 25 atoms) per primitive cell. 15R unit cell possesses an intermediate structure between an ideal cubic (c) and hexagonal (h) structures with a layer stacking sequence of $(\mathrm{chhh})_{3}$ or in other words, atomic stacking of "ABCBACABACBAACB". The corner-shared connectivity (cubic to hexagonal stacking ratio) is $20 \%$. It has $\mathrm{Mn}_{5} \mathrm{O}_{18}$ units (of five face-shared octahedra) connected to each other directly at their corners, forming a chain along the $c$-axis (Figure 1a). The crystal phase and corresponding unit cell parameters for the synthesized sample are obtained from Rietveld refinement of the room temperature x-ray diffraction patterns using High-score Plus software. The refinement indicates that the synthesized compound is in 15R type hexagonal phase possessing R-3m (No. 166) space group with rhombohedral symmetry. No additional or unexpected reflections could be identified thus confirming that the synthesized powder is of single-phase and free from any detectable impurity. The refined lattice parameters at room temperature are $\mathrm{a}=\mathrm{b}=5.6817 \AA, \mathrm{c}=35.369 \AA, \alpha=\beta=90^{\circ}$, and $\gamma=120^{\circ}$ which are comparable to the previous reports $[15,25,26]$. Three irreducible sites each for $\mathrm{Ba}, \mathrm{Mn}$, and $\mathrm{O}$ atoms in the unit cell which are labelled as Ba1, Ba2, Ba3, Mn1, Mn2, Mn3, and O1, O2, O3, respectively, are shown in Figure 1(a). The bond lengths and angles are listed in Table I. The refined crystallographic Wyckoff positions for each atom are listed in Table S1 in the supplemental material [27]. The Mn-Mn distance along the face-shared octahedra (2.4178 $2.4783 \AA)$ is comparable or smaller than the metallic $\gamma$-Mn $(2.47 \AA)$ which plays an important role in the physical properties of $\mathrm{BaMnO}_{3}$ [28]. The strong electrostatic repulsion between $\mathrm{Mn}$ atoms leads to distortion in face-shared octahedra making them asymmetric while cornershared octahedra remain symmetric with all the six Mn-O bonds of equal lengths (Table I) [15]. The spin arrangement in the magnetically ordered phase is displayed in Figure 1(b) which implies that the spins orient along the $a$-axis and are antiferromagnetically ordered along the $c$-axis [15] which will be discussed later. TEM images also confirm the formation of the lattice structure of the compound (Figure 1(c)). The $d$-spacing and lattice parameters are estimated and found to be in agreement with our PXRD results. Energy dispersive X-ray (EDAX) measurements were carried out for elemental analysis, details of which are given in Figure 1(d) and Table II. We found no detectable impurity elements or additional phases in the sample [see supplemental material for further details [27]].

To investigate the spin-ordering transitions, temperature-dependent magnetization measurements were carried out in two different protocols: zero-field cooling (ZFC) and field cooling (FC) under the applied field of 500 Oe as shown in Figure 2a. The transition

temperatures can be clearly determined from the inflection in the derivative, $\frac{d M(T)}{d T}$, shown in the inset of Figure 2a. Our data show two magnetic transitions: one as an anomaly at 330 ( \pm $0.2) \mathrm{K}$ and another at $230( \pm 0.2) \mathrm{K}$ which are, respectively, the outcomes of a short-ranged direct exchange interaction $\left(\mathrm{J}_{\mathrm{D}}\right)$ between $\mathrm{Mn}^{+4}$ ions in face-shared $\mathrm{MnO}_{6}$ octahedra of the $\mathrm{Mn}_{5} \mathrm{O}_{18}$ units and linear $\mathrm{Mn}-\mathrm{O}-\mathrm{Mn}$ superexchange $(\mathrm{J} 1)$ between $\mathrm{Mn}^{+4}$ ions located in corner- 
shared octahedra [29]. The bond angles for Mn2-O2-Mn3 and Mn3-O3-Mn1 are 81.07 and $78.28^{\circ}$, respectively, where the overlapping between $3 \mathrm{~d}$ orbitals of $\mathrm{Mn}^{+4}$ and $\mathrm{O}-2 \mathrm{p}$ orbitals is minimal, resulting in a relatively weak and negligible ferromagnetic (FM) exchange interaction which are denoted by $\mathrm{J} 2$ and J3, respectively (Figure 1b). Notably, the heat capacity as a function of temperature (Figure 2b) shows two anomalies (kinks) at $\sim 330( \pm 0.2) \mathrm{K}$ and $\sim 230$ $( \pm 0.2) \mathrm{K}$, corresponding to the two magnetic transitions thus corroborating our magnetization results. The observed low-temperature magnetic transition at $\sim 43( \pm 0.2) \mathrm{K}$ is associated with spin canting (See Figures S1-S4 in section SM2 of Supplemental material [27] for further details including field-dependent magnetization results and further analysis on magnetic transitions) [30, 31]. Below $230 \mathrm{~K}\left(\mathrm{~T}_{\mathrm{N}}\right)$, the spins orient along the $a$-axis and are antiferromagnetically ordered along the $c$-axis exhibiting an in-plane parallel arrangement with no direct exchange pathways, as shown in Figure 1b [spin alignment in full unit cell is shown in Figure S2 supplemental material [27]]. To understand the correlation between the phonons and magnetoelectric order parameters, we have performed a systematic Raman spectroscopic measurement with varying temperature and magnetic field as discussed below.

\section{Raman spectroscopy:}

According to group theory, the $15 \mathrm{R}$ structure with the space group R-3m leads to 18 Raman active and 27 IR active phonons at the $\Gamma$ point of the Brillouin zone with irreducible representations $\Gamma_{\text {Raman }}=8 A_{1 g}+10 E_{g}$ and $\Gamma_{I R}=12 A_{2 u}+15 E_{u}$, respectively [Tables III \& $\mathrm{S} 2]$. Because of the site symmetries, the atoms Ba1, Mn1, and O1 participate only in IR active vibrations and are not involved in Raman active modes, whereas, $\mathrm{Ba} 2, \mathrm{Ba} 3, \mathrm{Mn} 2, \mathrm{Mn} 3, \mathrm{O} 2$, and $\mathrm{O} 3$ take part in both IR and Raman active vibrations. Figure 3(a) shows the Raman spectra collected at room temperature $(300 \mathrm{~K})$ and $80 \mathrm{~K}$ along with their Lorentzian fits. The symmetry assignment of the modes is done based on our polarization-dependent Raman spectra (Figure S5 in supplemental material [27]), SYMMODES-Bilbao Crystallographic Server [32], and previous reports [26,33]. The spectrum at $80 \mathrm{~K}$ presents an additional number of modes as compared to the one collected at $300 \mathrm{~K}$, implying the possible association with a change in the crystallographic and/or magnetic symmetry of $\mathrm{BaMnO}_{3}$ at low temperatures [34-37]. To get a better understanding of the phonons, we have collected Raman spectra systematically in the temperature range of $80-800 \mathrm{~K}$ and analysed their evolution by fitting with Lorentzian multifunction. The Raman spectra at a few typical temperatures are shown in Figure S6 (Supplemental material [27]). The phonon parameters (Spectral weight/intensity, frequency, and linewidth) as a function of temperature for a few selected modes are displayed in Figure 3. The spectral weight (intensity) decreases with increasing temperature for almost all the modes, possibly because the temperature-dependence of intensity due to Bose factor is masked by the temperature-dependence of the absorption coefficient (intensity of the other modes is shown in Figure S7 in the supplemental material [27]) [38]. The frequency and linewidth of the majority of the phonons show the usual anharmonic trend (i.e. decrease (increase) in mode frequency (linewidth) with increasing temperature) as shown in Figure S8 and S9 in the supplemental material [27]. Notably, these modes exhibit a change in slope at one of the magnetic transition temperatures $\left(\mathrm{T}_{\mathrm{N}}\right.$ or $\left.\mathrm{T}_{\mathrm{S}}\right)$ or at a temperature $\left(\mathrm{T}_{\mathrm{D}}\right) \sim 280 \mathrm{~K}$. More importantly, five of the phonon modes (namely, P7, P8, P12, P14, and P15) are observed to respond drastically at these temperatures as shown in Figure 3. The mode $\mathrm{P} 7$ at $\sim 390 \mathrm{~cm}^{-1}$ vanishes right above $\mathrm{T}_{\mathrm{D}} \sim 280$ 
$\mathrm{K}$. We attribute the origin of $\mathrm{P} 7$ mode to the displacement of $\mathrm{Mn}^{+4}$ ions along the $c$-axis amounting to a change in local symmetry that may be associated with the dielectric anomaly reported in ref. [15]. The frequency (of mode P7) shows an anomalous temperature-dependence (i.e. a decrease in frequency with decreasing temperature) while the linewidth shows an extremely large broadening (by almost 4-fold) in the temperature range of 80-280 K. The anomalies in P7 could be related to spin-phonon coupling to be discussed later. On the other hand, the P12 and P14 modes appear right below the magnetic transitions $\mathrm{T}_{\mathrm{N}}$ and $\mathrm{T}_{\mathrm{S}}$, respectively. In addition, each of the modes at $\sim 415 \mathrm{~cm}^{-1}$ (P8) and $\sim 653 \mathrm{~cm}^{-1}$ (P15) clearly split into two modes right below $\mathrm{T}_{\mathrm{N}} \sim 230( \pm 0.2) \mathrm{K}$. To note that our temperature-dependent $\mathrm{x}$-ray diffraction measurements (to be discussed later) show no clear signatures of structural phase transition in the investigated temperature range $(90-400 \mathrm{~K})$ thus ruling out structural phase transition as the possible origin for the splitting of the two modes P8 and P15. However, possibilities of further changes in the local symmetries at $\mathrm{T}_{\mathrm{N}}$ arising from additional displacements of Mn ions cannot be completely ruled out. Further, the mode P6 deviates from the anharmonic trend (discussed below) at temperatures below $\mathrm{T}_{\mathrm{N}}$ (similar to the deviations seen for the other modes below $\mathrm{T}_{N} / \mathrm{T}_{\mathrm{S}} / \mathrm{T}_{\mathrm{D}}$, shown in Figures $\mathrm{S} 8$ and $\mathrm{S} 9$ [27]). These observations suggest the presence of a strong correlation between phonons and magnetoelectric parameters in $15 \mathrm{R}-\mathrm{BaMnO}_{3}$. In order to understand the origin of phonon anomalies and appearance of new peaks in Raman spectra below magnetic transitions, we have analysed the temperature-dependence of phonon frequency and linewidth using the anharmonic model.

In general, the temperature-dependence of a phonon frequency can be written as [39-42]:

$$
\omega(T)=\omega_{a n h}(T)+\Delta \omega_{e l-p h}(T)+\Delta \omega_{s p-p h}(T)
$$

The first term $\omega_{\text {anh }}(T)$ is the anharmonic contribution as discussed below. The contribution to the change in phonon frequency due to electron-phonon coupling is given by the term $\Delta \omega_{e l-p h}(T)$ which is absent in $15 \mathrm{R}-\mathrm{BaMnO}_{3}$ due to its insulating nature. The renormalization of the frequency due to spin-phonon coupling is accounted by $\Delta \omega_{s p-p h}(T)$. In the cubic anharmonic process, the phonon of frequency $\omega_{0}$ decays into two phonons of equal frequency $\omega_{0} / 2$ satisfying the energy and momentum conservation. The temperature-dependence of the phonon frequency due to cubic anharmonicity (three-phonon process) is given by [39,40]:

$$
\omega_{a n h}(T)=\omega_{0}+A\left[1+\frac{2}{\left(e^{\frac{\hbar \omega_{0}}{2 k_{B} T}}-1\right)}\right]
$$

Similarly, the temperature-dependence of phonon linewidth due to cubic anharmonicity can be written as:

$$
\Gamma_{a n h}(T)=\Gamma_{0}+C\left[1+\frac{2}{\left(e^{\frac{\hbar \omega_{0}}{2 k_{B} T}}-1\right)}\right]
$$

where $\omega_{0}$ and $\digamma_{0}$ are frequency and linewidth of the phonon at absolute zero temperature, $A$, and $C$, are cubic anharmonic coefficients for frequency and linewidth, respectively, $\hbar$ is 
reduced Planck constant, $k_{B}$ is Boltzmann constant and $T$ is the variable temperature. As seen in our data, both the frequency and the linewidth of almost all phonons show a finite deviation from the anharmonic temperature-dependence, indicating the possible magnetostriction in the system (Figure 3(c, d) \& Figures S8, S9 [27]). The solid lines in Figure 3 (c, d) represent the fitting with Eq. 2 and 3. The mode P12 appears below $\mathrm{T}_{\mathrm{N}}$ (origin to be discussed later) and its frequency and linewidth exhibit the expected thermal behaviour (Eq. 2 and 3, respectively). Most importantly, the mode $\mathrm{P} 7$ that appears below $\mathrm{T}_{\mathrm{D}}$ shows an anomalous shift in frequency while its linewidth undergoes an anomalous broadening which cannot be explained by anharmonicity alone. These indicate a strong role of spin-phonon coupling. Further, the P6 and P14 modes show a deviation from anharmonic thermal behaviour in their temperaturedependence of frequency and linewidth at low temperatures below the Neel temperature $\mathrm{T}_{\mathrm{N}} \sim$ $230 \mathrm{~K}$. In the absence of any structural phase transition, we attribute the observed anomalies in P6, P7, and P14 phonons to the spin-phonon coupling. The deviation in frequency $\left(\Delta \omega_{s p-p h}\right)$ in modes P6, P7, and P14 from their anharmonic behaviours (see Figure 3(c)) with respect to temperature are captured in Figure 4. The strength of spin-phonon coupling is estimated using mean-field and two-spin cluster approximations as explained below.

\section{Spin-phonon coupling:}

In magnetic materials, when a phonon is coupled to the spin degrees of freedom, its frequency gets renormalized in proportion to the nearest-neighbour spin-spin correlation function < $S_{i} . S_{j}>$ (Schematically shown in Figure S10 in the supplemental material [27]) which can be written as [41-43]:

$$
\Delta \omega_{s p-p h}=-\lambda_{s p}<S_{i} . S_{j}>=-\lambda_{s p} \Phi(T) S^{2}
$$

where $\lambda_{s p}$ is the strength of spin-phonon coupling and $\Phi$ is the short-range order parameter. Lockwood et al. [42] theoretically estimated the $\Phi(T)$ for $S=2\left(\mathrm{FeF}_{2}\right)$ and $S=5 / 2\left(\mathrm{MnF}_{2}\right)$ antiferromagnetic systems using mean-field and two-spin cluster approximations. Since the estimates of $\Phi$ do not vary much with the value of the spin $(S)$, it was also used reasonably for $S=1\left(\mathrm{NiF}_{2}\right.$ and $\left.\mathrm{NiO}\right)$ antiferromagnets $[41,43]$. Therefore, it is also reasonable to make use of the $\Phi$ estimated by Lockwood et al. [42] for $\mathrm{BaMnO}_{3}$ in our case where $S=3 / 2\left(\mathrm{Mn}^{4+}\right)$. Thus, the spin-phonon coupling can be estimated using the relation [43]:

$$
\lambda_{s p}=-\frac{\omega\left(T_{\text {Low }}\right)-\omega_{a n h}\left(T_{\text {Low }}\right)}{\left[\Phi\left(T_{\text {Low }}\right)-\Phi\left(2 T_{N}\right)\right] S^{2}}
$$

where $\omega\left(T_{\text {Low }}\right)$ is the experimental phonon frequency at the lowest temperature recorded $\left(T_{\text {Low }} \sim 80 \mathrm{~K}\right.$ in our case) while the $\omega_{\text {anh }}\left(T_{\text {Low }}\right)$ is the corresponding anharmonic estimate of the phonon frequency at the same temperature. The obtained values for the spin-phonon coupling $\left(\lambda_{s p}\right)$ for the modes P6, P7, and P14 using eqn. (5) are 1.2, 3.8, and $1.5 \mathrm{~cm}^{-1}$, respectively. The coupling strength for the modes is reasonably strong and may be compared with some of the reported values of the strength in compounds like $9 \mathrm{R}-\mathrm{BaMnO}_{3}(\lambda \sim 0.5$ to 3.4 $\left.\mathrm{cm}^{-1}[44]\right), 4 \mathrm{H}-\mathrm{Sr}_{0.6} \mathrm{Ba}_{0.4} \mathrm{MnO}_{3}\left(\lambda \sim 2.2 \mathrm{~cm}^{-1}[45]\right), \mathrm{MnF}_{2}\left(\lambda \sim 0.4 \mathrm{~cm}^{-1}[42]\right), \mathrm{FeF}_{2}(\lambda \sim 1.3$ $\left.\mathrm{cm}^{-1}[42]\right), \mathrm{La}_{2} \mathrm{CoMnO}_{6}\left(\lambda \sim 1.7-2.1 \mathrm{~cm}^{-1}[46], \operatorname{Pr}_{2} \mathrm{CoMnO}_{6}\left(\lambda \sim 0.51-1.61 \mathrm{~cm}^{-1}[47,48]\right)\right.$, 
$\mathrm{Cr}_{2} \mathrm{Ge}_{2} \mathrm{Te}_{6}\left(\lambda \sim 0.24-1.2 \mathrm{~cm}^{-1}[49]\right), \mathrm{Sr}_{2} \mathrm{CoO}_{4}\left(\lambda \sim 3.5 \mathrm{~cm}^{-1}[50]\right), \mathrm{NiO}\left(\lambda \sim-7.9 \mathrm{~cm}^{-1}\right.$ and $14.1 \mathrm{~cm}^{-1}$ for TO and LO phonons, respectively [43]), $\mathrm{ZnCr}_{2} \mathrm{O}_{4}\left(\lambda \sim 3.2-6.2 \mathrm{~cm}^{-1}\right.$ [51]), $\mathrm{NaOsO}_{3}\left(\lambda \sim 40 \mathrm{~cm}^{-1}[52]\right)$, and $\mathrm{CuO}\left(\lambda \sim 50 \mathrm{~cm}^{-1}[53]\right)$.

\section{Emergence of new Raman modes: Effect of magnetostriction}

Emergence of new modes in Raman spectra is possible below magnetic transition due to various reasons such as concurrent magnetic and structural phase transition [54], spinexcitations including one-magnon or two-magnon Raman processes [35, 55], or magnetostriction [36,37]. Since phonons and magnetic excitations exhibit contrasting behaviour under external magnetic fields, we have investigated magnetic-field-dependence of the Raman spectrum to shed light on the origin of the new modes. $15 \mathrm{R}-\mathrm{BaMnO}_{3}$ was cooled down to $4 \mathrm{~K}$ and the magnetic field was applied in the range of 0-9T. Figure 5a compares the Raman spectra at 0 T, $3 \mathrm{~T}, 6 \mathrm{~T}$, and $9 \mathrm{~T}$ where no major effect on the spectrum is visible, thus we can rule out the magnetic excitation as the origin for the new modes and assign them as new phonon modes. Figure $5 \mathrm{~b}$ shows the frequency of a few selected phonons as a function of the magnetic field (see also Figures S11-S14 in the supplemental material [27] for phonon parameters). The frequency of all the phonons shows a decreasing trend with increasing magnetic field thus suggesting a lattice expansion under an external magnetic field, indicating a possible role of magnetostriction.

In order to ensure any possible role of structural phase transition, on the origin of the new phonon modes, we have performed temperature-dependent powder x-ray diffraction measurements (PXRD). Figure 6a displays the PXRD patterns collected at a few temperatures in the range of 90-400 K. The number of reflections in the PXRD pattern remains the same in the entire investigated temperature range and Rietveld analysis of the data confirms the absence of structural phase transition.

In absence of a structural phase transition, the origin of the new phonon modes below the magnetic transition temperatures could be attributed to the local changes in the symmetry arising from the displacement of Mn-ions at low temperatures. The thermal expansion is a key parameter to ascertain the magnetostriction because it involves the coupling between magnetic and elastic degrees of freedom. We have analysed the unit cell parameters (lattice parameters and unit cell volume) as a function of temperature. Figure $6 \mathrm{~b}$ shows the lattice parameters and unit cell volume as a function of temperature in the range of 90-450 K (bond lengths are given in Figure S15 in supplemental material [27]). The 15R-BaMnO 3 expands upon heating and shows positive thermal expansion. In general, the temperature-dependent lattice parameters due to thermal expansion can be written as [56]:

$$
a(T)=a_{0}\left[1+\frac{b e^{\frac{d}{T}}}{T\left(e^{\frac{d}{T}}-1\right)^{2}}\right] \quad \text { and } \quad c(T)=c_{0}\left[1+\frac{f e^{\frac{g}{T}}}{T\left(e^{\frac{g}{T}}-1\right)^{2}}\right]
$$

where, $a_{0}$ and $\mathrm{c}_{0}$ are the in-plane and out-of-plane lattice constants at $0 \mathrm{~K}$, whereas $\mathrm{b}, \mathrm{d}, \mathrm{f}$, and $\mathrm{g}$ are fitting parameters. Similarly, unit cell volume as a function of temperature can be expressed as: 


$$
V(T)=V_{0}\left[1+\frac{A}{\left(e^{\frac{\theta}{T}}-1\right)}\right]
$$

where, $V_{0}$ is cell volume extrapolated to $0 \mathrm{~K}, \theta$ is the Debye temperature, and $A$ is a fitting parameter (see Table S4 and Section SM7 in the supplemental material [27]). Importantly, the temperature-dependence of the lattice parameters and the volume show a deviation at $T_{S}$ as shown in Figure 6. As discussed earlier, a short-ranged magnetic ordering occurs below $\mathrm{T}_{\mathrm{S}} \sim$ $330 \mathrm{~K}$ that changes the trend of lattice expansion owing to local symmetry changes (without undergoing a structural phase transition) thus corroborating the presence of magnetostriction in $15 \mathrm{R}-\mathrm{BaMnO}_{3}$ [57].

It is to be noted that the frequency of a phonon $\omega \propto \sqrt{k}(k=$ spring constant) where $k$ decreases with increasing unit cell volume (bond length). The decrease in phonon frequency with increasing magnetic field, as shown in Figure 5b, signify an expansion of the unit cell volume under magnetic field further suggesting the presence of magnetostriction in $15 \mathrm{R}-\mathrm{BaMnO}_{3}$. Here, we have quantified the magnetostriction in two ways: (1) Using thermal expansion ( $\mathrm{x}-$ ray diffraction measurements) in the absence of external magnetic field and (2) using lattice expansion (Raman spectroscopic measurements) under external magnetic field at a constant temperature.

If the spontaneous distortion in the unit cell (without changing the crystal symmetry) due to magnetic ordering is large enough, it can be detected by $\mathrm{x}$-ray diffraction technique which also provides several advantages over conventional strain-gauge and capacitive techniques [57-59]. The spontaneous volume magnetostriction $\left(\lambda_{m s}^{V}(T)\right)$ at a given temperature ' $T$ ' (below $\mathrm{T}_{\mathrm{N}}$ ) can be defined as [61]:

$$
\lambda \lambda_{m s}^{V}(T)=\frac{V_{A F M}(T)-V_{P M}(T)}{V_{P M}(T)}
$$

where $V_{A F M}(T)$ is the actual unit cell volume at the temperature $T$ in the antiferromagnetic phase whereas $V_{P M}(T)$ is the hypothetical volume of the unit cell if it were in the paramagnetic (nonmagnetic) phase at the same temperature. The value of $\lambda_{m s}^{V}$ at $90 \mathrm{~K}$ (lowest measured temperature) is $\sim 32( \pm 7) \times 10^{-4}$, which is comparable to the reported value of magnetostriction in the spinel compounds $\mathrm{Zn}_{1-\mathrm{x}} \mathrm{Cu}_{\mathrm{x}} \mathrm{Cr}_{2} \mathrm{Se}_{4}$ (4.6 to $24.9 \times 10^{-4}$ at $100 \mathrm{~K}$ for varying $\mathrm{x}$ ) measured by XRD technique [61] (temperature-dependent values of $\lambda_{m s}^{V}$ for $15 \mathrm{R} \mathrm{BaMnO}_{3}$ are given in Figure S16 in the supplemental material [27]).

On the other hand, magnetostriction can also be defined as the change in the dimensions of a magnetic material under an external magnetic field. It is often characterized by $\frac{\Delta l}{l}$ where $l$ is the original physical length of the material and $d l$ is the change in the dimension of the material under the applied magnetic field $(H)$ [60]. It can be expressed as $\lambda_{m s}^{l}=\frac{\Delta l}{l} \approx \frac{l(H)-l(0)}{l(0)}$ for the linear magnetostriction and similarly, the volume magnetostriction is $\lambda_{m s}^{V}=\frac{\Delta V}{V} \approx \frac{V(H)-V(0)}{V(0)}$, where, for our case we have considered the unit cell dimensions instead of the physical dimensions. The change in unit cell volume can be related to the corresponding change in 
phonon frequency through Grüneisen parameter as $\frac{\Delta \omega}{\omega}=\gamma \frac{\Delta V}{V}$. In solids, the typical value of mode Grüneisen parameter $(\gamma)$ is $\sim 1$ (see supplemental material [27] for details). Under the assumption of $\gamma \sim 1$, the volume magnetostriction of $15 \mathrm{R}-\mathrm{BaMnO}_{3}$ at $4 \mathrm{~K}$ is estimated to be in the range of 0.01 to $5.6 \times 10^{-4}$ (Figure $\mathrm{S} 21$ in supplementary material [27]), which is comparable to the range of reported magnetostriction values $\left(10^{-3}\right.$ to $\left.10^{-6}\right)$ in various systems [62-68] and also comparable to the value obtained through $\mathrm{x}$-ray diffraction technique discussed above (see Figure S17-21 and Table S5 in the section SM8 of Supplemental material [27] for details on estimation of $\gamma$ and magnetostriction in $15 \mathrm{R}-\mathrm{BaMnO}_{3}$ ).

\section{Conclusions}

In summary, we have synthesized polycrystalline hexagonal $\mathrm{BaMnO}_{3}$ in $15 \mathrm{R}$ phase using the solid-state reaction route. A new phonon of frequency $\sim 390 \mathrm{~cm}^{-1}(\mathrm{P} 7)$, associated with $\mathrm{Mn}$ vibrations, appears below $\sim 280 \mathrm{~K}$ (due to local distortions arising from the displacement of Mn-ions) and exhibits anomalous behaviour due to a strong spin-phonon coupling $\left(\lambda_{\text {sp-ph }} \sim 3.8\right.$ $\mathrm{cm}^{-1}$ ). Additionally, several new modes appear below the magnetic transition temperatures (330 $\mathrm{K}$ and $230 \mathrm{~K}$ ) due to the local changes in symmetry arising from the displacement of Mn-ions. Further, we have observed evidences of magnetostriction in $15 \mathrm{R}-\mathrm{BaMnO}_{3}$ through a change in lattice expansion rate across the magnetic ordering temperature in XRD measurements and signatures of lattice expansion under applied magnetic field in Raman scattering. We have shown that the lattice vibrations are strongly correlated with the electric and magnetic properties in $15 \mathrm{R}-\mathrm{BaMnO}_{3}$. We believe that the spin-phonon coupling and lattice strain (magnetostriction) can be the new parameters to tune the magnetoelectric properties of the system thus making it potential for near room-temperature technological applications and motivating further experimental and theoretical studies.

\section{Acknowledgement}

Authors acknowledge IISER Bhopal for research facilities, B. P. acknowledges the University Grant Commission for fellowship and S. S. acknowledges DST/SERB (project No's ECR/2016/001376 and CRG/2019/002668) and Nanomission (Project No. SR/NM/NS$84 / 2016(C)$ ) for research funding. Support from DST-FIST (Project No. SR/FST/PSI195/2014(C) is also thankfully acknowledged. Authors acknowledge Mr. Sajilesh K P (Department of Physics, IISER Bhopal) for helping with the heat capacity measurements.

\section{References}

1. J. Orenstein and A. J. Millis, "Advances in the Physics of High-Temperature Superconductivity", Science 288, 468 (2000).

2. Eerenstein W, Mathur ND, Scott JF. "Multiferroic and magnetoelectric materials", Nature 442, 759 (2006).

3. Ideue T, Kurumaji T, Ishiwata S, Tokura Y, "Giant thermal Hall effect in multiferroics" Nat. Mater. 16, 797 (2017).

4. Khomskii, D. I., \& Sawatzky, G. A. "Interplay between spin, charge and orbital degrees of freedom in magnetic oxides", Solid state communications 102, 87 (1997). 
5. Sebastian F. Maehrlein, Ilie Radu, Pablo Maldonado, Alexander Paarmann, Michael Gensch, Alexandra M. Kalashnikova, Roman V. Pisarev, Martin Wolf, Peter M. Oppeneer, Joseph Barker, Tobias Kampfrath, "Dissecting spin-phonon equilibration in ferrimagnetic insulators by ultrafast lattice excitation”, Sci Adv. 4, eaar5164 (2018).

6. Moya X, Mathur ND, “Thermal hall effect: Turn your phonon”, Nat. Mater. 16, 784 (2017).

7. Manuel Bibes and Agnès Barthélémy, "Multiferroics: Towards a magnetoelectric memory", Nature Materials 7, 425 (2008).

8. T. Jungwirth, X. Marti, P. Waldley, J. Wunderlich, “Antiferromagnetic spintronics”, Nat. Nanotechnol. 11, 231 (2016).

9. T. Jungwirth, J. Sinova, A. Manchon, X. Marti, J. Wunderlich and C. Felser, "The multiple directions of antiferromagnetic spintronics", Nat. Phys. 14, 200 (2018).

10. P. Němec, M. Fiebig, T. Kampfrath and A. V. Kimel, "Antiferromagnetic optospintronics", Nat. Phys. 14, 229 (2018).

11. June Hyuk Lee, Lei Fang, Eftihia Vlahos, Xianglin Ke, Young Woo Jung, Lena Fitting Kourkoutis, Jong-Woo Kim, Philip J. Ryan, Tassilo Heeg, Martin Roeckerath, Veronica Goian, Margitta Bernhagen, Reinhard Uecker, P. Chris Hammel, Karin M. Rabe, Stanislav Kamba, Jürgen Schubert, John W. Freeland, David A. Muller, Craig J. Fennie, Peter Schiffer, Venkatraman Gopalan, Ezekiel Johnston-Halperin \& Darrell G. Schlom1, "A strong ferroelectric ferromagnet created by means of spin-lattice coupling", Nature 466, 954 (2010).

12. Masahito Mochizuki, Nobuo Furukawa, and Naoto Nagaosa, "Theory of spin-phonon coupling in multiferroic manganese perovskites $\mathrm{RMnO}_{3}$ ”, Phys. Rev. B 84, 144409 (2011).

13. Carlos Calero, E. M. Chudnovsky, and D. A. Garanin, "Field Dependence of the Electron Spin Relaxation in Quantum Dots”, Phys. Rev. Lett. 95, 166603 (2005).

14. Daniel Khomskii, "Classifying multiferroics: Mechanisms and effects", Physics 2, 20 (2009).

15. O B Korneta, T F Qi, M Ge, S Parkin, L E De Long, P Schlottmann and G Cao, "Correlated giant dielectric peaks and antiferromagnetic transitions near room temperature in pure and alkali-doped $\mathrm{BaMnO}_{3-\delta}$ ”, J. Phys.: Condens. Matter 23, 435901 (2011).

16. Terutaro Nakamura, "Soft phonon in $\mathrm{BaTiO}_{3}$ ", Journal of Ferroelectrics 137, 65 (1992).

17. Y Luspint, J L Servoin and F Gervais, "Soft mode spectroscopy in barium titanate", J. Phys. C: Solid St. Phys., 13,3761 (1980).

18. Bommareddy Poojitha, Km Rubi, Soumya Sarkar, R. Mahendiran, T. Venkatesan, and Surajit Saha, "Effect of phonon anharmonicity on ferroelectricity in $\mathrm{Eu}_{\mathrm{x}} \mathrm{Ba}_{1-\mathrm{x}} \mathrm{TiO}_{3}$ ", Physical Review Materials 3, 024412 (2019).

19. T. A. Tyson, T. Wu, K. H. Ahn, S.-B. Kim, and S.-W. Cheong, "Local spin-coupled distortions in multiferroic hexagonal $\mathrm{HoMnO}_{3}$ ", Physical Review B 81, 054101 (2010).

20. T. E. Saunders and J. T. Chalker, "Structural phase transitions in geometrically frustrated antiferromagnets", Phys. Rev. B 77, 214438 (2008).

21. I. P. Handayani, A. A. Nugroho, S. Riyadi, G. R. Blake, N. Mufti, T. T. M. Palstra, and P. H. M. van Loosdrecht, "Correlation between lattice vibrations with charge, orbital, 
and spin ordering in the layered manganite $\operatorname{Pr}_{0.5} \mathrm{Ca}_{1.5} \mathrm{MnO}_{4}$ ", Physical Review B 92 , 205101 (2015).

22. A. Nonato, B. S. Araujo, A. P. Ayala, A. P. Maciel, S. Yanez-Vilar, M. SanchezAndujar, M. A. Senaris-Rodriguez, and C. W. A. Paschoal1 "Spin-phonon and magnetostriction phenomena in $\mathrm{CaMn}_{7} \mathrm{O}_{12}$ helimagnet probed by Raman spectroscopy", Appl. Phys. Lett. 105, 222902 (2014).

23. V. G. Sathe, S. Tyagi, and G. Sharma, "Electron-phonon coupling in perovskites studied by Raman Scattering”, Journal of Physics: Conference Series 755, 012008 (2016).

24. K. Momma and F. Izumi, VESTA 3 for three-dimensional visualization of crystal, volumetric and morphology data, J. Appl. Cryst. 44, 1272 (2011).

25. J.J. Adkin, M.A. Hayward, " $\mathrm{BaMnO}_{3-\mathrm{x}}$ revisited: a structural and magnetic study", Chem. Mater. 19, 755(2007).

26. D.P. Kozlenko, N.T. Dang, T.L. Phan, S.E. Kichanov, L.H. Khiem, S.G. Jabarov, T.A. Tran, T.V. Manh, A.T. Le, T.K. Nguyen, B.N. Savenko, "The structural, magnetic and vibrational properties of Ti-doped $\mathrm{BaMnO}_{3}$ ”, Journal of Alloys and Compounds 695, 2539 (2017).

27. See Supplemental Material for additional data and information about the magnetic and heat capacity measurements, assignment of modes, polarization-, temperature-, and magnetic field-dependent Raman spectra, and powder XRD data.

28. Richard J.D. Tilley, "Perovskites, Structure-Property Relationships, first ed.", Wiley, UK, 2016.

29. P. D. Battle, T. C. Gibb, and C. W. Jones, "The structural and magnetic properties of $\mathrm{SrMnO}_{3}$ : A reinvestigation", Journal of Solid State Chemistry 74, 60 (1988).

30. L. Ghivelder, I. Abrego Castillo, N. McN. Alford, G.J. Tomka, P.C. Riedi, J. Mac Manus-Driscoll, A. K. M. Akther Hossain, L.F. Cohen, "Specific heat of La1xCax $\mathrm{MnO}_{3-\mathrm{d}}$ ", J. Mag. Mag. Mat. 189, 274 (1998).

31. B.F. Woodfield, M.L. Wilson, J.M. Byers, "Low-Temperature Specific Heat of La1${ }_{x} \mathrm{Sr}_{\mathrm{x}} \mathrm{MnO}_{3+\mathrm{d}}$ ", Phys. Rev. Lett. 78, 3201(1997).

32. E. Kroumova, M.I. Aroyo, J.M. Perez-Mato, A. Kirov, C. Capillas, S. Ivantchev, H. Wondratschek, "Bilbao crystallographic server: useful databases and tools for phasetransition studies", Phase Trans. 76, 155(2003).

33. Chen Xiang-Bai, Hien Nguyen Thi Minh, YANG In-Sang, LEE Daesu, NOH Tae-Won "A Raman Study of the Origin of Oxygen Defects in Hexagonal Manganite Thin Films", Chin. Phys. Lett. 29, 126103 (2012).

34. Robert A. Evarestov and Andrei V. Bandura, "First-Principles Calculations on the Four Phases of $\mathrm{BaTiO}_{3}$ ", Journal of Computational Chemistry, 33, 1123 (2012).

35. Paul A. Fleury, "Paramagnetic Spin Waves and Correlation Functions in $\mathrm{NiF}_{2}$ ", Phys. Rev. 180, 591 (1969).

36. H.C. Hsu, F.C. Chou, K. Koyama, K. Watanabe, H.L. Liu, "Spin-phonon coupling in antiferromagnetic $\mathrm{Bi}_{2} \mathrm{Sr}_{2} \mathrm{CoO}_{6+\delta}$ : an infrared reflectance study", Phys. Rev. B 79, 155109(2009). 
37. C. Toulouse, C. Martin, M-A. Measson, Y. Gallais, A. Sacuto, and M. Cazayous, "Magnetic transitions in $\mathrm{CaMn}_{7} \mathrm{O}_{12}$ : Raman observation of spin-phonon couplings", Phys. Rev. B 99, 024303 (2019).

38. Shuibo Xie, Enrique Iglesia, and Alexis T. Bell, "Effects of Temperature on the Raman Spectra and Dispersed Oxides", J. Phys. Chem. B 105, 5144 (2001).

39. M. Balkanski, R.F. Wallis, E. Haro, "Anharmonic effects in light scattering due to optical phonons in silicon”, Phys. Rev. B 28, 1928(1983).

40. P.G. Klemens, "Anharmonic decay of optical phonons”, Phys. Rev. 148, 845 (1966).

41. D.J. Lockwood, "Spin-Phonon interaction and mode softening in $\mathrm{NiF}_{2}$ ", Low Temp. Phys. 28, 505 (2002).

42. D. J. Lockwood, M.G. Cottam, "The spin-phonon interaction in $\mathrm{FeF}_{2}$ and $\mathrm{MnF}_{2}$ studied by Raman spectroscopy”, J. Appl. Phys. 64, 5876(1988).

43. E. Atyan, B. Debnath, F. Kargar, Y. Barlas, M.M. Lacerda, J.X. Li, R.K. Lake, J. Shi, A.A. Balandin, "Spin-phonon coupling in antiferromagnetic nickel oxide", Appl. Phys. Lett. 111,252402 (2017).

44. Bommareddy Poojitha, Anjali Rathore, and Surajit Saha "Spin-phonon coupling in $\mathrm{Sr}$ and $\mathrm{Ti}$ incorporated 9R-BaMnO 3 " Journal of Magnetism and Magnetic Materials 483,212 (2019).

45. R. Rawat, D.M. Phase, R.J. Choudhary, "Spin-phonon coupling in hexagonal $\mathrm{Sr}_{0.6} \mathrm{Ba}_{0.4} \mathrm{MnO}_{3}$ ", J. Magn. Magn. Mater. 441,398 (2017).

46. Ch. Meyer, V. Roddatis, P. Ksoll, B. Damaschke, V. Moshnyaga, "Structure, magnetism, and spin-phonon coupling in heteroepitaxial $\mathrm{La}_{2} \mathrm{CoMnO}_{6} / \mathrm{Al}_{2} \mathrm{O}_{3}(0001)$ films", Phys. Rev. B 98,134433 (2018).

47. Dhirendra Kumar, Satish Kumar, Vasant G. Sathe, "Spin-phonon coupling in ordered double perovskites $\mathrm{A}_{2} \mathrm{CoMnO}_{6}(\mathrm{~A}=\mathrm{La}, \mathrm{Pr}, \mathrm{Nd})$ probed by micro-Raman spectroscopy", Solid State Commun. 194,59 (2014).

48. Changzheng Xie, Lei Shi, Jiyin Zhao, Shiming Zhou, Yang Li, Xueyou Yuan, "Spinphonon coupling in $\mathrm{R}_{2} \mathrm{CoMnO}_{6}(\mathrm{R}=\mathrm{Pr}, \mathrm{Nd}, \mathrm{Sm})$ thin films under biaxial compressive strain”, J. Appl. Phys. 120,155302 (2016).

49. Yao Tian, Mason J. Gray, Huiwen Ji, R.J. Cava, Kenneth S. Burch, "Magneto-elastic coupling in a potential ferromagnetic 2D atomic crystal", 2D Mater. 3,025035 (2016).

50. P.K. Pandey, R.J. Choudhary, D.K. Mishra, V.G. Sathe, D.M. Phase, "Signature of spin-phonon coupling in $\mathrm{Sr}_{2} \mathrm{CoO}_{4}$ thin film: a Raman spectroscopic study", Appl. Phys. Lett. 102, 142401 (2013).

51. A.B. Sushkov, O. Tchernyshyov, W. Ratcliff, Cheong, H.D. Drew, "Probing spin correlations with phonons in the strongly frustrated magnet $\mathrm{ZnCr}_{2} \mathrm{O}_{4}$ ", Phys. Rev. Lett. 94,137202 (2005).

52. S. Calder, et al., "Enhanced spin-phonon-electronic coupling in a 5d oxide", Nat. Commun. 6,8916 (2015).

53. X.K. Chen, J.C. Irwin, J.P. Franck, "Evidence for a strong spin-phonon interaction in cupric oxide", Phys. Rev. B 52, R13130(R) (1995).

54. T.N. Stanislavchuk, A.P. Litvinchuk, Rongwei Hu, Young Hun Jeon, Sung Dae Ji, S.W. Cheong, A.A. Sirenko, "Optical properties, lattice dynamics, and structural phase transition in hexagonal 2H-BaMnO 3 single crystals”, Phys. Rev. B 92, 134308 (2015). 
55. P. A. Fleury, S. P. S. Porto, L. E. Cheesman, and H. J. Guggenheim, "Light scattering by spin waves in $\mathrm{FeF}_{2}$ " Phys. Rev. Lett. 17, 84 (1966).

56. Charles Kittel, "Introduction to Solid State Physics", seventh ed., Wiley, New York, 2003.

57. F.J. Darnell, "Temperature Dependence of Lattice Parameters for Gd, Dy, and Ho", Phys. Rev. 130, 1825 (1963).

58. F.J. Darnell, "Magnetostriction in Dysprosium and Terbium”, Phys. Rev. 132, 128 (1963).

59. J. Samuel Smart and Selma Greenwald, "Crystal Structure Transitions in Antiferromagnetic Compounds at the Curie Temperature", Phys. Rev. 82, 113 (1951).

60. B. K. Tanner, "Diffraction techniques in the study of magnetic domains and magnetostriction", Sci. Prog., Oxf. 67, 411 (1981).

61. J. Kusz, S. Juszczyk and J. Warczewski, "An X-ray diffraction study of magnetostriction in $\mathrm{Zn}_{1-\mathrm{x}} \mathrm{Cu}_{\mathrm{x}} \mathrm{Cr}_{2} \mathrm{Se}_{4}(0 \cdot 2<\mathrm{x}<1 \cdot 0)$ ", J. Appl. Cryst. 21, 898(1988).

62. P. N. Anantharamaiah and P. A. Joy, "Enhancing the strain sensitivity of $\mathrm{CoFe}_{2} \mathrm{O}_{4}$ at low magnetic fields without affecting the magnetostriction coefficient by substitution of small amounts of Mg for Fe", Phys. Chem. Chem. Phys., 18, 10516 (2016).

63. M. Doerr, M. Rotter and A. Lindbaum, "Magnetostriction in rare-earth based antiferromagnets", Advances in Physics 54, 1 (2005).

64. S. S. Fonton and A. V. Zalesskiu, "Magnetostriction of a single crystal of hexagonal ferrite $\mathrm{BaFe}_{18} \mathrm{O}_{27}$ ", J. Exptl. Theoret. Phys. (U.S.S.R.) 47, 1693 (1964).

65. E. Klokholm and J. Aboaf, "The saturation magnetostriction of thin polycrystalline films of iron, cobalt, and nickel” Journal of Applied Physics 53, 2661 (1982).

66. Abhishek Majumder, Kodam Ugendar, Anoop Baby K. B., Venkatarao Chunchu, R. A. Mondal and G. Markandeyulu, "Anisotropy, Magnetostriction and Converse Magnetoelectric effect in Dy substituted Ni Ferrite", Physics Procedia 75, 238 (2015).

67. A. B. Smith and R. V. Jones, "Magnetostriction in Nickel Ferrite and Cobalt-Nickel Ferrite" Journal of Applied Physics 37, 1001 (1966).

68. J. Smit and H. P. J. Wijn, Ferrites, Philips Technical Library, Eindhoven, The Netherlands, 1959. 
Table I. Lattice parameters, bond lengths, and bond angles of $15 \mathrm{R}-\mathrm{BaMnO}_{3}$ obtained from the Rietveld refinement of $\mathrm{x}$-ray diffraction data at room temperature.

\begin{tabular}{|l|l|l|}
\hline $\begin{array}{l}\text { Lattice } \\
\text { parameters }\end{array}$ & $\begin{array}{l}\mathrm{a}=\mathrm{b}=5.6817 \AA, \mathrm{c}=35.369 \AA, \alpha=\beta= \\
90^{\circ}, \gamma=120^{\circ}\end{array}$ \\
\hline \multirow{3}{*}{ Bond lengths } & $\mathrm{Mn} 2-\mathrm{Mn} 2$ & $3.8406 \AA$ \\
\cline { 2 - 3 } & $\mathrm{Mn} 2-\mathrm{Mn} 3$ & $2.4783 \AA$ \\
\cline { 2 - 3 } & $\mathrm{Mn} 3-\mathrm{Mn} 1$ & $2.4178 \AA$ \\
\cline { 2 - 3 } & $\mathrm{Mn} 2-\mathrm{O} 1$ & $1.9203 \AA$ \\
\cline { 2 - 3 } & $\mathrm{Mn} 2-\mathrm{O} 2$ & $1.9294 \AA$ \\
\cline { 2 - 3 } & $\mathrm{Mn3-O} 2$ & $1.8834 \AA$ \\
\cline { 2 - 3 } & $\mathrm{Mn} 3-\mathrm{O} 3$ & $1.9248 \AA$ \\
\cline { 2 - 3 } & $\mathrm{Mn} 1-\mathrm{O} 3$ & $180^{\circ}$ \\
\hline Bond angles & $\mathrm{Mn} 1-\mathrm{O} 1-\mathrm{Mn} 1$ & $78.28^{\circ}$ \\
\cline { 2 - 3 } & $\mathrm{Mn} 2-\mathrm{O} 2-\mathrm{Mn} 3$ & \\
\cline { 2 - 3 } & $\mathrm{Mn} 3-\mathrm{O} 3-\mathrm{Mn} 1$ & \\
\hline
\end{tabular}

Table II. EDAX data of $15 \mathrm{R}-\mathrm{BaMnO}_{3}$.

\begin{tabular}{|l|l|l|}
\hline \multicolumn{3}{|c|}{ EDAX results } \\
\hline Element & Weight\% & Atomic\% \\
\hline O K & $22.00( \pm 0.08)$ & $63.47( \pm 0.09)$ \\
\hline $\mathrm{Mn} \mathrm{K}$ & $20.43( \pm 0.13)$ & $17.17( \pm 0.13)$ \\
\hline $\mathrm{Ba} \mathrm{L}$ & $57.57( \pm 0.14)$ & $19.36( \pm 0.06)$ \\
\hline Total & 100.00 & 100 \\
\hline
\end{tabular}


Table III. Raman active phonons in $15 \mathrm{R}-\mathrm{BaMnO}_{3}$.

\begin{tabular}{|c|c|c|c|c|}
\hline \multicolumn{2}{|c|}{$\begin{array}{c}\text { Space group R-3m } \\
\text { (No.166) } \\
\text { and } \\
\digamma_{\text {Raman }}=8 A_{1 g}+10 E\end{array}$} & \multicolumn{2}{|c|}{$\begin{array}{l}\text { 6c (Ba2, Ba3) } \\
\text { 6c (Mn2, Mn3) } \\
18 \mathrm{~h}(\mathrm{O} 2, \mathrm{O} 3)\end{array}$} & $\begin{array}{c}\frac{\text { Irreducible }}{\text { representations }} \\
2 A_{1 g}+2 E_{g} \\
2 A_{1 g}+2 E_{g} \\
4 A_{1 g}+6 E_{g}\end{array}$ \\
\hline \multirow{2}{*}{ Mode } & \multirow{2}{*}{ Symmetry } & \multicolumn{2}{|c|}{$\omega\left(\mathrm{cm}^{-1}\right)$} & Atoms involved in \\
\hline & & at $80 \mathrm{~K}$ & at $300 \mathrm{~K}$ & the vibration \\
\hline P1 & $E_{\mathrm{g}}$ & $82.0( \pm 0.1)$ & $80.9( \pm 0.1)$ & $\mathrm{Ba}$ \\
\hline $\mathrm{P} 2$ & $\overline{A_{1 \mathrm{~g}}}$ & $100.7( \pm 0.1)$ & $99.3( \pm 0.1)$ & $\mathrm{Ba}$ \\
\hline P3 & $E_{\mathrm{g}}$ & $176.5( \pm 0.1)$ & $174.7( \pm 0.1)$ & $\mathrm{Ba}$ \\
\hline $\mathrm{P} 4$ & $\bar{E} E_{\mathrm{g}}$ & $241.8( \pm 0.1)$ & $238.9(0.1)$ & $\mathrm{Mn}$ \\
\hline $\mathrm{P} 5$ & $\overline{E \mathrm{~g}}$ & $278.5( \pm 0.1)$ & $275.6( \pm 0.1)$ & $\mathrm{Mn}$ \\
\hline P6 & $E_{\mathrm{g}}$ & $361.4( \pm 0.1)$ & $358.4( \pm 0.1)$ & $\mathrm{Mn}$ \\
\hline P7 & $A_{1 \mathrm{~g}}$ & $\begin{array}{c}383.5( \pm 0.2) \\
(\text { Appears at } \mathrm{T}<280 \mathrm{~K})\end{array}$ & Absent & $\mathrm{Mn}$ \\
\hline P8A & $A_{1 \mathrm{~g}}$ & $418.2( \pm 0.1)$ & & \\
\hline P8B & $A_{1 \mathrm{~g}}$ & $\begin{array}{c}422.0( \pm 0.1) \\
(\text { Appears at } \mathrm{T}<230 \mathrm{~K})\end{array}$ & $( \pm 0.1)\left(E_{\mathrm{g}}\right)$ & $\mathrm{O}$ \\
\hline P9 & $E_{\mathrm{g}}$ & $430.3( \pm 0.1)$ & $427.5( \pm 0.1)$ & $\mathrm{O}$ \\
\hline P10 & $A_{1 \mathrm{~g}}$ & $535.6( \pm 0.4)$ & $530.0( \pm 0.4)$ & $\mathrm{O}$ \\
\hline P11 & $E_{\mathrm{g}}$ & $557.9( \pm 0.1)$ & $552.2( \pm 0.1)$ & $\mathrm{O}$ \\
\hline P12 & $E_{\mathrm{g}}$ & $\begin{array}{c}565.9( \pm 0.1) \\
(\text { Appears at } \mathrm{T}<230 \mathrm{~K})\end{array}$ & Absent & $\mathrm{O}$ \\
\hline P13 & $E_{\mathrm{g}}$ & $583.0( \pm 0.1)$ & $578.8( \pm 0.1)$ & $\mathrm{O}$ \\
\hline P14 & $A_{1 \mathrm{~g}}$ & $643.2( \pm 0.1)$ & $636.9( \pm 0.3)$ & $\mathrm{O}$ \\
\hline $\mathrm{P} 15 \mathrm{~A}$ & $A_{1 \mathrm{~g}}$ & $655.8( \pm 0.1)$ & & \\
\hline P15B & $A_{1 \mathrm{~g}}$ & $\begin{array}{c}659.9( \pm 0.4) \\
(\text { Appears at } \mathrm{T}<230 \mathrm{~K})\end{array}$ & $( \pm 0.1)\left(E_{\mathrm{g}}\right)$ & $\mathrm{O}$ \\
\hline
\end{tabular}




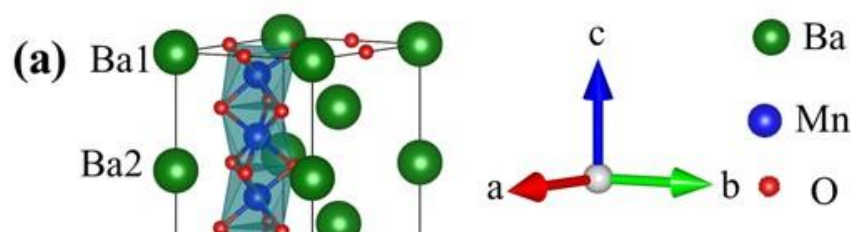

(c)

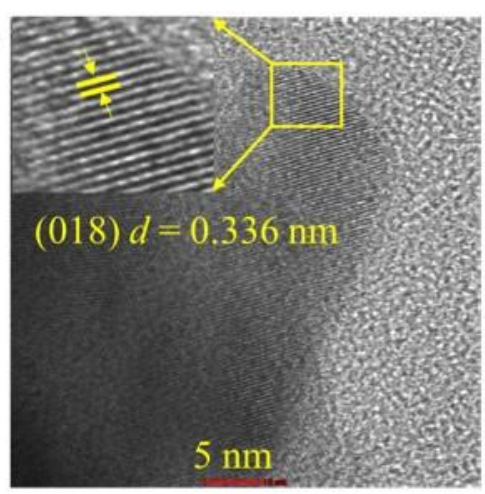

(b)
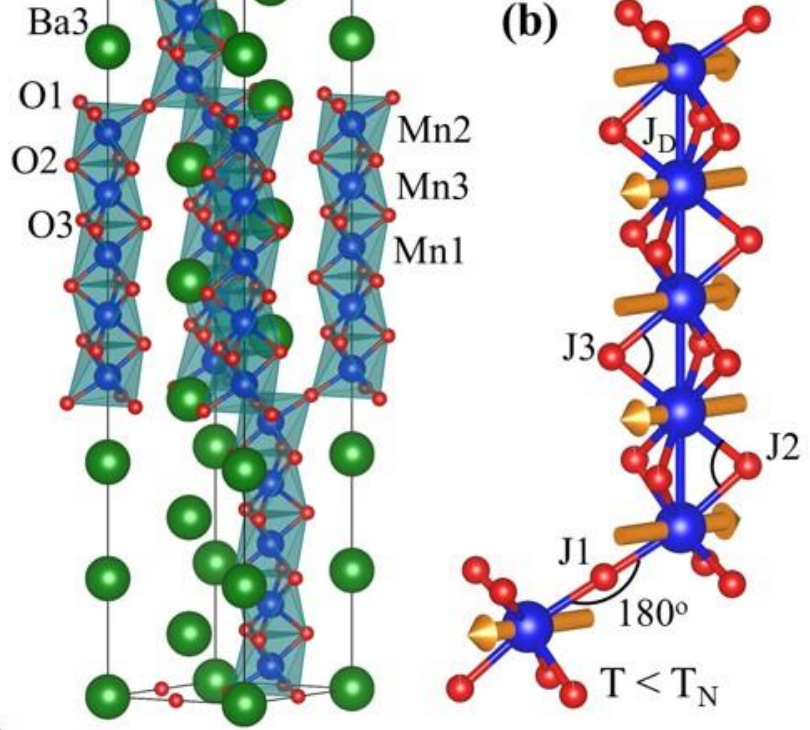

(d)

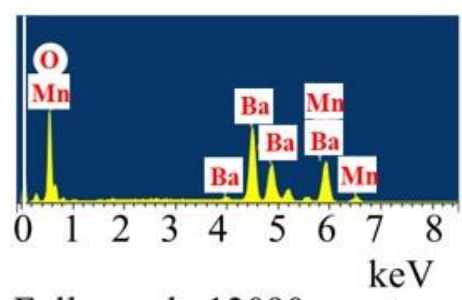

Full y-scale 13090 counts

Cursor: $0.000 \mathrm{keV}$

(e)

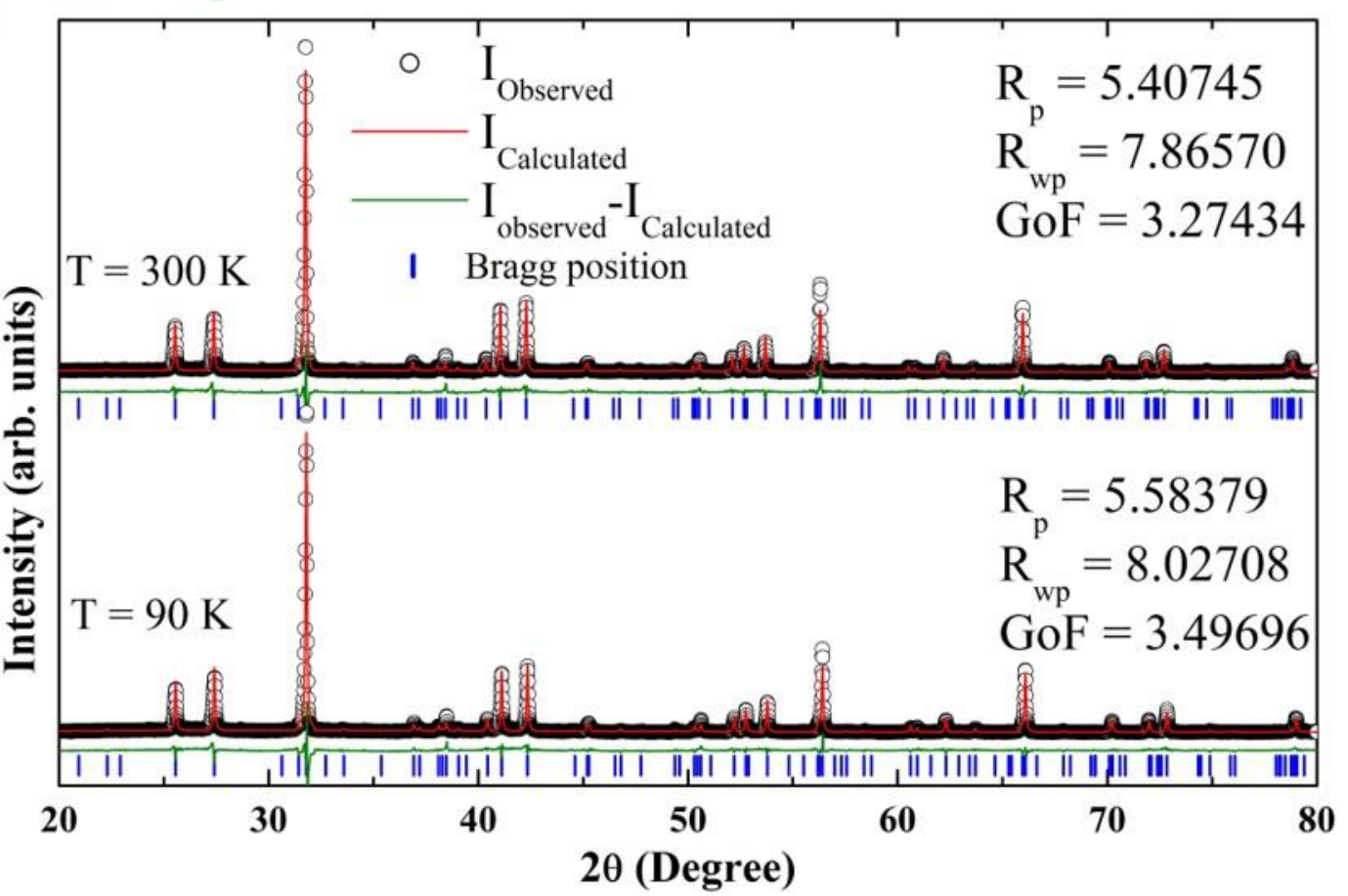

Figure 1. (Colour online) (a) Crystallographic unit cell of 15R-BaMnO3, (b) spin arrangement of Mn-ions in antiferromagnetic phase, (c) TEM image showing the lattice planes, (d) EDAX spectrum showing the stoichiometric presence of the elements, and (e) $X$-ray diffraction patterns collected at $300 \mathrm{~K}$ and $90 \mathrm{~K}$. R-factors $\left(R_{p}\right.$ and $\left.R_{w p}\right)$ and the Goodness of Fit (GoF) obtained from the refinements are also given. 


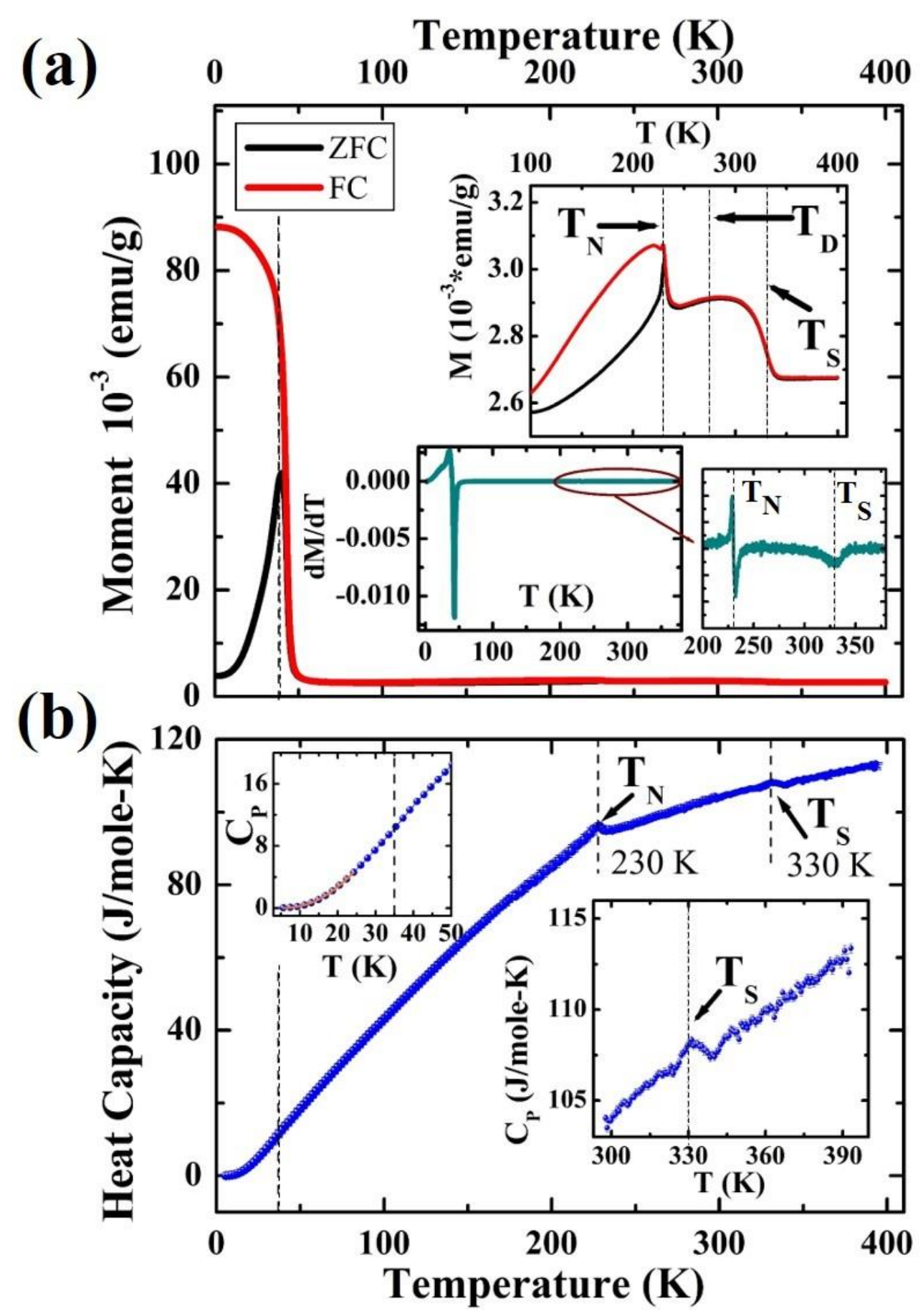

Figure 2. (Colour online) (a) Magnetic moment as a function of temperature and insets show enlarged view in high temperature range and the temperature derivative of magnetization to clearly identify the antiferromagnetic transition temperatures, $(b)$ temperature-dependent heat capacity, left inset is enlarged view of the heat capacity $\left(C_{P}\right)$ in the low temperature range (the orange solid line is a fit with $C_{P}(T)=\beta T^{3}+\delta T^{2}$ (eqn. S2) explained in supplementary material suggesting A-type or canted antiferromagnetic ordering at these temperatures) and right inset is to clearly show the response at $T_{S} \sim 330 \mathrm{~K}$. 
(a)

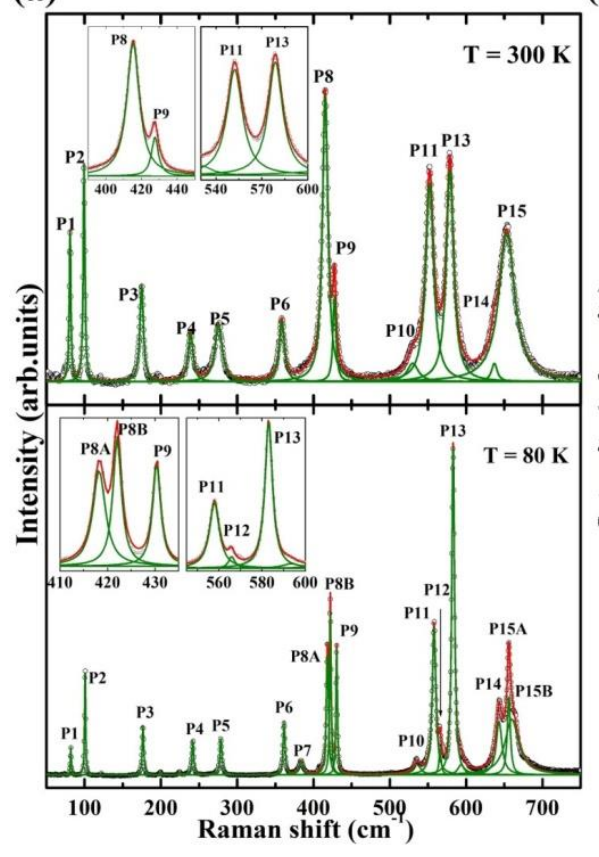

(b)

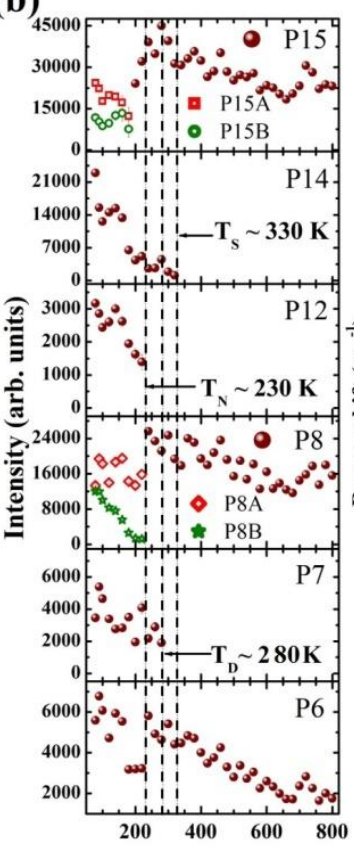

(c)

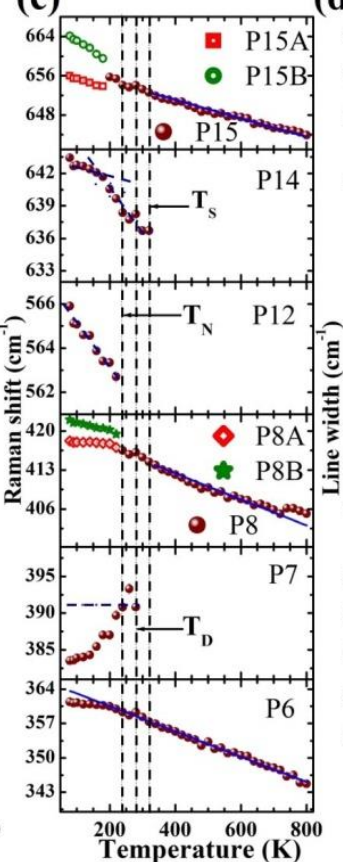

(d)

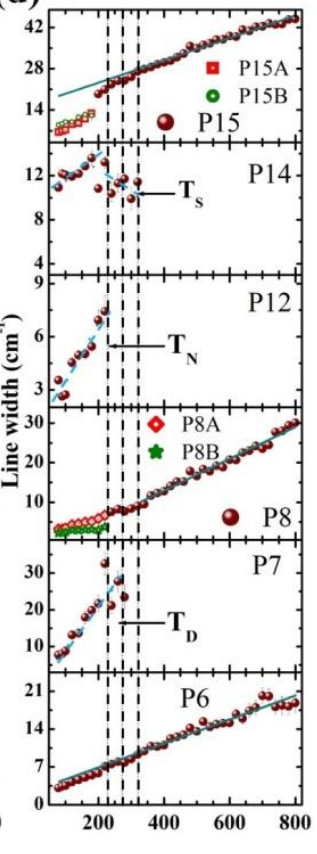

Figure 3. (Colour online) (a) Raman spectra of $15 \mathrm{R}-\mathrm{BaMnO}_{3}$ collected at $80 \mathrm{~K}$ and $300 \mathrm{~K}$, (b) spectral weight (intensity), (c) frequency, and (d) linewidth of selected phonons are plotted against temperature. Solid lines in $(c)$ and $(d)$ represent the fitting with anharmonic model (Eq. 2 and 3 in text) whereas the dashed lines for modes P12 and P14 below $T_{N}$ are guide to eye. Error bars are smaller than or comparable to the symbol size.

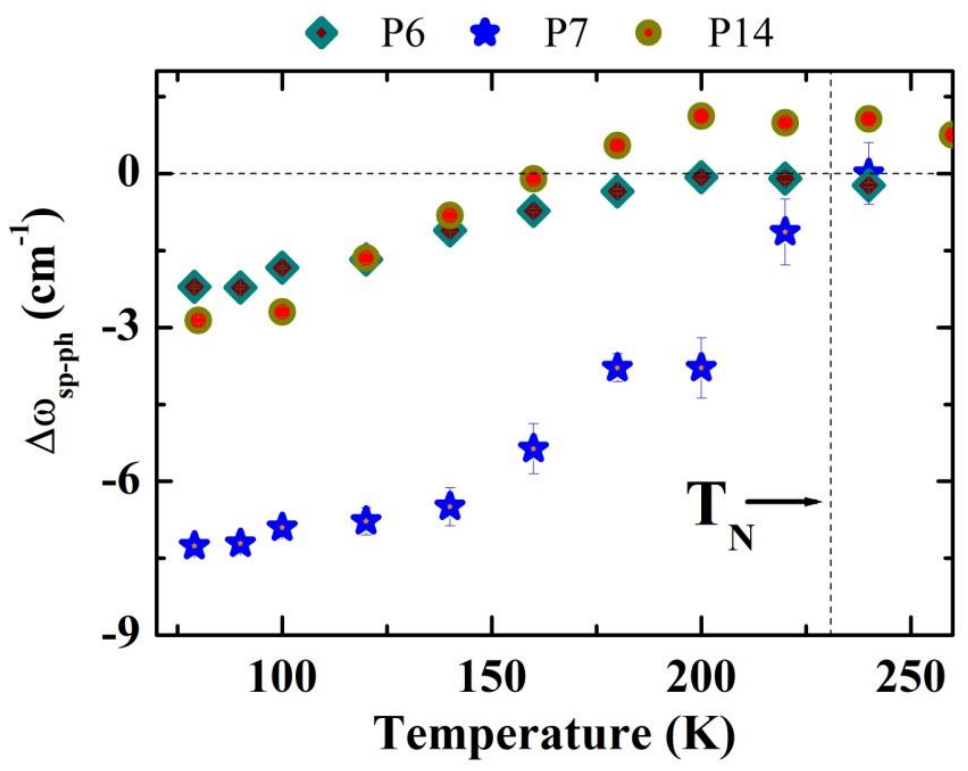

Figure 4. (Colour online) (a) The deviation of phonon frequency from anharmonic behaviour $\left(\Delta \omega_{s p-p h}\right)$ as a function of temperature (see text). Error bars are within or comparable to the symbol size. 
(a)

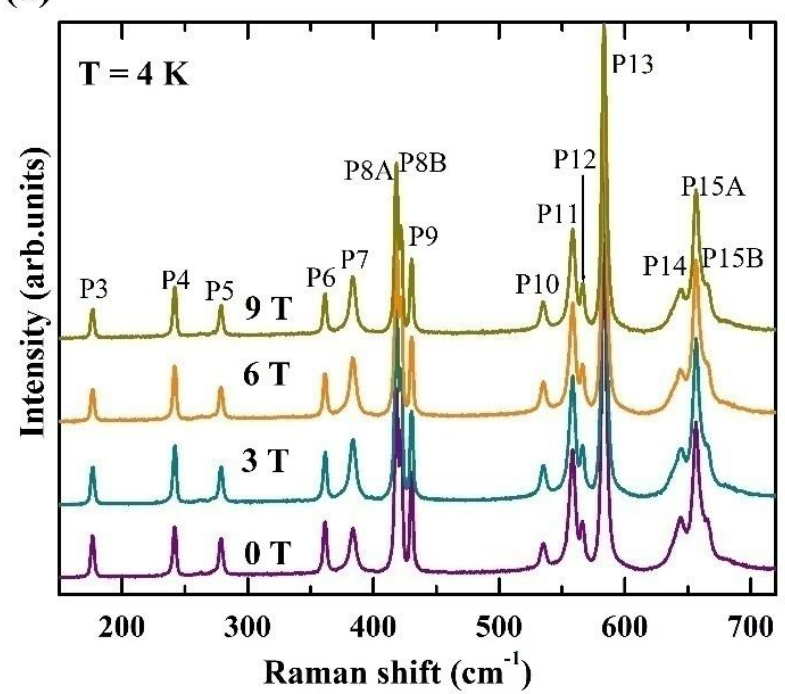

(b)

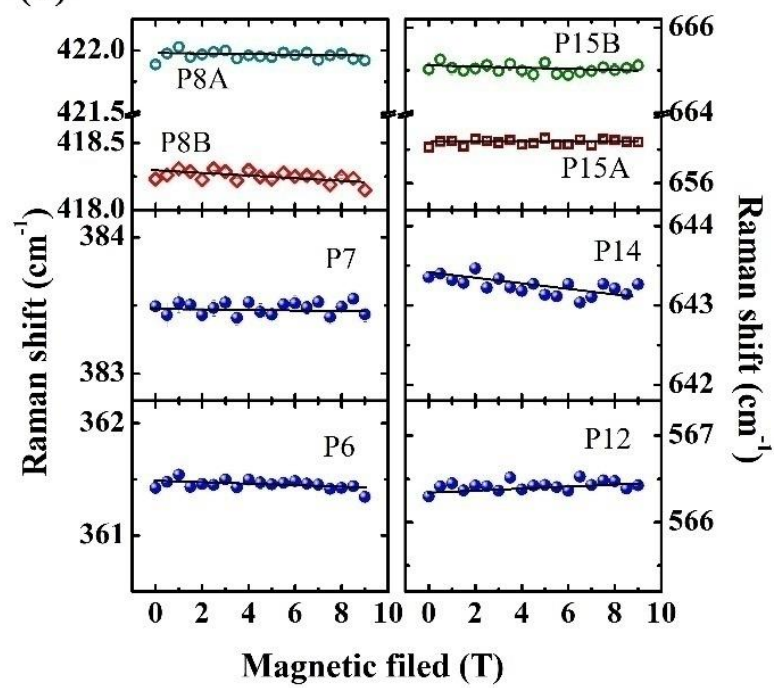

Figure 5. (Colour online) (a) Raman spectra of 15R-BaMnO $\mathrm{O}_{3}$ collected under a few applied magnetic fields at $4 \mathrm{~K}$, (b) frequency of selected phonons are plotted as a function of magnetic field, solid lines are guide to eye showing a decreasing trend with increasing field. Error bars are smaller than the symbol size. 

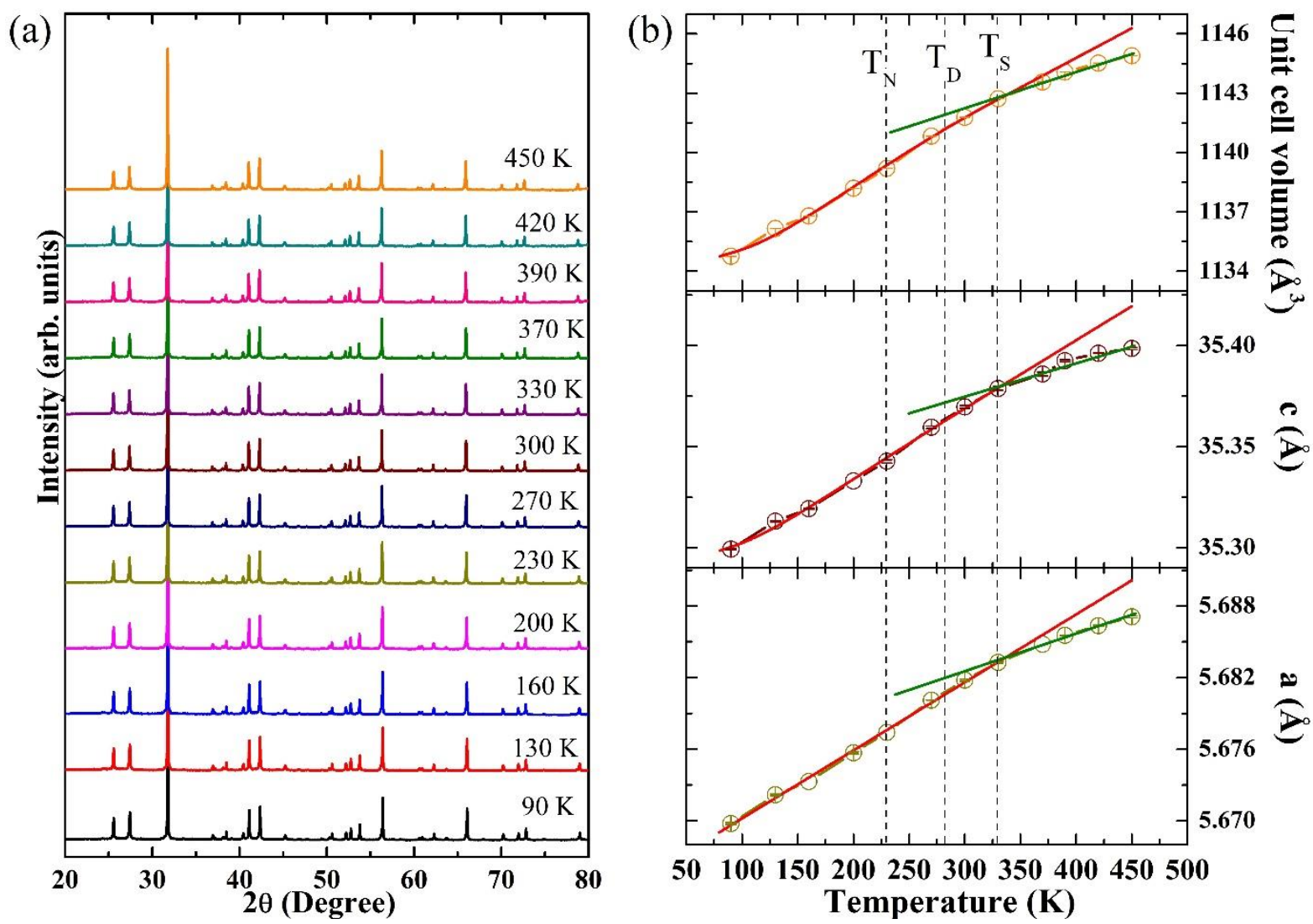

Figure 6. (Colour online) (a) X-ray diffraction patterns collected at a few temperatures and (b) unit cell parameters as a function of temperature, red solid lines represent fittings presenting the expansion (eq. 6 and 7 explained in text), green solid lines are also fit with Eq. 6 and 7 showing the deviation from expected behaviour. Error bars in (b) are within the symbol size. 


\title{
Supplemental material
}

\section{Signatures of magnetostriction and spin-phonon coupling in magnetoelectric hexagonal 15R-BaMnO 3}

\author{
Bommareddy Poojitha, Anjali Rathore, Ankit Kumar, and Surajit Saha* \\ Department of Physics, Indian Institute of Science Education and Research, Bhopal 462066, India \\ *Correspondence: surajit@iiserb.ac.in
}

This supplemental material file contains additional data and information about the magnetic and heat capacity measurements, assignment of modes, polarization-, temperature-, and magnetic field-dependent Raman spectra, and powder XRD data. The figures and respective details are given below.

\section{SM1: Structural details from $x$-ray diffraction}

The Wyckoff positions for each atom extracted from Rietveld refinement are listed in Table $\mathrm{S} 1$. Results indicate the oxygen vacancies at $\mathrm{O} 2$ site. The amount of oxygen deficiency $(\sim 0.01)$ is similar to the value expected for $15 \mathrm{R}$ structure [1].

Table S1. Rietveld refined Wyckoff positions of atoms in $15 \mathrm{R}-\mathrm{BaMnO}_{3}$ unit cell.

\begin{tabular}{|c|c|c|c|c|c|}
\hline atom & site & $\mathbf{x}$ & $\mathbf{y}$ & $\mathbf{z}$ & F.O \\
\hline $\mathrm{Ba} 1$ & $3 \mathrm{a}$ & 0 & 0 & $1 / 2$ & 1 \\
\hline $\mathrm{Ba} 2$ & $6 \mathrm{c}$ & 0 & 0 & 0.36611 & 1 \\
\hline $\mathrm{Ba} 3$ & $6 \mathrm{c}$ & 0 & 0 & 0.23464 & 1 \\
\hline $\mathrm{Mn} 1$ & $3 \mathrm{a}$ & 0 & 0 & 0 & 1 \\
\hline $\mathrm{Mn} 2$ & $6 \mathrm{c}$ & 0 & 0 & 0.13843 & 1 \\
\hline $\mathrm{Mn} 3$ & $6 \mathrm{c}$ & 0 & 0 & 0.06836 & 1 \\
\hline $\mathrm{O} 1$ & $9 \mathrm{~d}$ & $1 / 2$ & 0 & $1 / 2$ & 1 \\
\hline $\mathrm{O} 2$ & $18 \mathrm{~h}$ & 0.51947 & 0.48053 & 0.23094 & $0.9875(1)$ \\
\hline $\mathrm{O} 3$ & $18 \mathrm{~h}$ & 0.51573 & 0.48427 & 0.36708 & 1 \\
\hline
\end{tabular}




\section{SM2: Magnetism and Specific heat}

Inverse susceptibility as a function of temperature of $15 \mathrm{R}-\mathrm{BaMnO}_{3}$ is shown in Figure $\mathrm{S} 1$ that exhibits non-Curie-Weiss behaviour above $\mathrm{T}_{\mathrm{S}}$; where, $\mathrm{M}(\mathrm{T})$ increases (inverse susceptibility decreases) slightly with increasing temperature till $400 \mathrm{~K}$, implying that there may be shortrange spin-spin correlations even in the paramagnetic phase. As the temperature is lowered below $\mathrm{T}_{\mathrm{S}}$, ZFC and FC curves in $\mathrm{M}(\mathrm{T})$ meet down to $\mathrm{T}_{\mathrm{N}}$ and show significant bifurcation below $\mathrm{T}_{\mathrm{N}}$, especially below $\mathrm{T}^{*} \sim 43( \pm 0.2) \mathrm{K}$ exhibiting a peak-like feature in ZFC curve. These signatures suggest that $15 \mathrm{R}-\mathrm{BaMnO}_{3}$ possibly undergoes a phase transition from antiferromagnetic (AFM) state to ferrimagnetic or canted AFM or a glassy state. The spin structure in antiferromagnetic phase is shown in Figure S2. To get further clarification about the nature of the observed magnetic transitions, we have collected magnetic hysteresis $\mathrm{M}(\mathrm{H})$ loops at a few typical temperatures and shown in Figure S3. The isothermal magnetization versus magnetic-field curves are linear passing through the origin throughout the temperature range down to $43 \mathrm{~K}$ which confirms the antiferromagnetic ordering in the system below $\mathrm{T}_{\mathrm{S}}$. Notably, clear hysteresis loops are observed below $\mathrm{T}^{*}$. The coercive field $\left(\mathrm{H}_{\mathrm{C}}\right)$ is found to decrease with increasing temperature and it vanishes above $\mathrm{T}^{*} \sim 43 \mathrm{~K}$ (inset of Figure S3). To be noted that the $\mathrm{M}(\mathrm{H})$ loops do not saturate even up to 7 Tesla of applied magnetic field which suggests that the ordering below $\mathrm{T}^{*}$ is either ferrimagnetic or canted AFM or spin glass. Importantly, a broad peak feature is observed in the first derivative of specific heat with respect to temperature thus suggesting that the magnetic ordering below $\mathrm{T}^{*}$ is of canted antiferromagnetic type (Figure S4) [2].

In general, the temperature-dependent heat capacity can be written as;

$$
C(T)=C_{\text {elect }}+C_{\text {latt }}+C_{\text {mag }}
$$

The term $C_{\text {elect }}=\gamma T$ is the electronic contribution, $C_{\text {latt }}=\beta T^{3}+\alpha T^{5}$ is the lattice contribution, while the $C_{m a g}$ is the magnetic contribution to the specific heat. $C_{m a g}=\delta T^{3 / 2}$ in case of a ferromagnet. For a standard Néel antiferromagnetically ordered phase, the magnetic contribution is proportional to $T^{3}$ which is similar to the Debye lattice term. However, for Atype antiferromagnetic order it can be written as $C_{\text {mag }}=\delta T^{2}[3,4]$. The specific heat at low temperatures (below $22 \mathrm{~K}$ ) is non-linear in temperature thus ruling out any electronic contribution due to the insulating nature of $15 \mathrm{R}-\mathrm{BaMnO}_{3}$.

As can be seen in Figure $2 \mathrm{~b}$ (left inset), the heat capacity data of $15 \mathrm{R}-\mathrm{BaMnO}_{3}$ in the low temperature region (below $22 \mathrm{~K}$ ) is analysed with the equation;

$$
C(T)=\beta T^{3}+\delta T^{2}
$$

A good fitting is obtained with $\mathrm{R}^{2}=0.996$ upon considering Eq. (S2). The $\delta T^{2}$ term is considered due to canted antiferromagnetic nature of $\mathrm{BaMnO}_{3}$ in the low temperature region. An addition of $T^{3 / 2}$ term does not appropriately fit the experimental data while addition of $\alpha T^{5}$ term does not improve the fitting either thus ruling out the contributions relevant to these terms. To be noted that the $T^{3}$ term is dominant below about $20 \mathrm{~K}$, as shown in Figure S4b. The 
obtained fitting parameters are $\beta=0.21 \pm 0.02 \frac{\mathrm{mJ}}{\mathrm{mol.} \mathrm{K}^{4}}$ and $\delta=2.45 \pm 0.60 \frac{\mathrm{mJ}}{\mathrm{mol} \cdot \mathrm{K}^{3}}$. The Debye temperature is extracted from $\beta$ value using the relation $\theta_{D}=\frac{12 \pi^{4} p R}{5 \beta}$; where $p$ is number of atoms per formula unit, and $\mathrm{R}$ is universal gas constant. The obtained value for Debye temperature is $\theta_{D} \sim 358 \pm 34 \mathrm{~K}$ which is in very good agreement with the value obtained from the temperature dependent $\mathrm{x}$-ray diffraction results $(\theta \sim 352 \pm 20 \mathrm{~K})$.

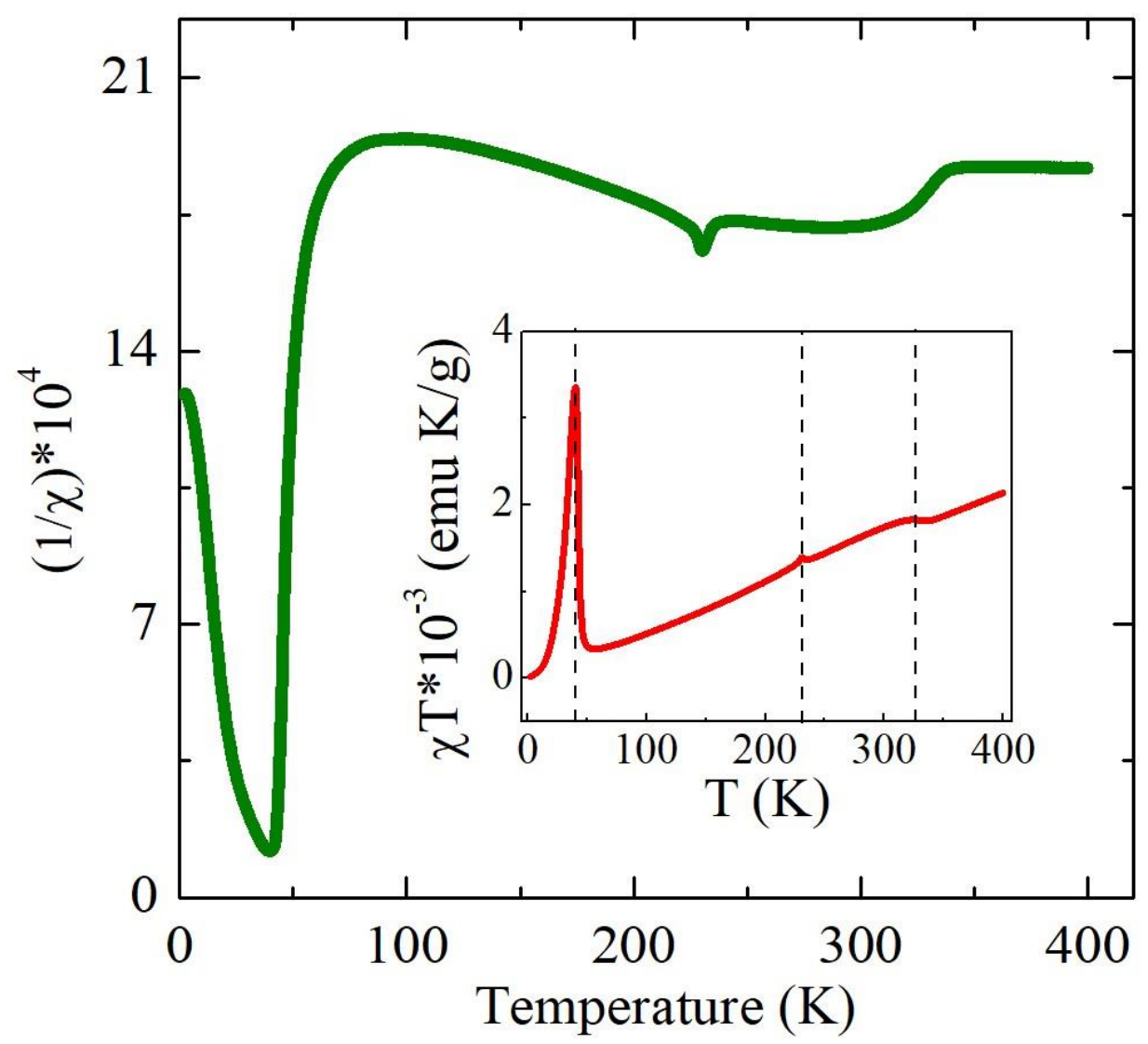

Figure S1. $\chi^{-1}$ vs $\mathrm{T}$, inset shows the plot of $\chi \mathrm{T}$ vs $\mathrm{T}$ for $15 \mathrm{R}-\mathrm{BaMnO}_{3}$. 


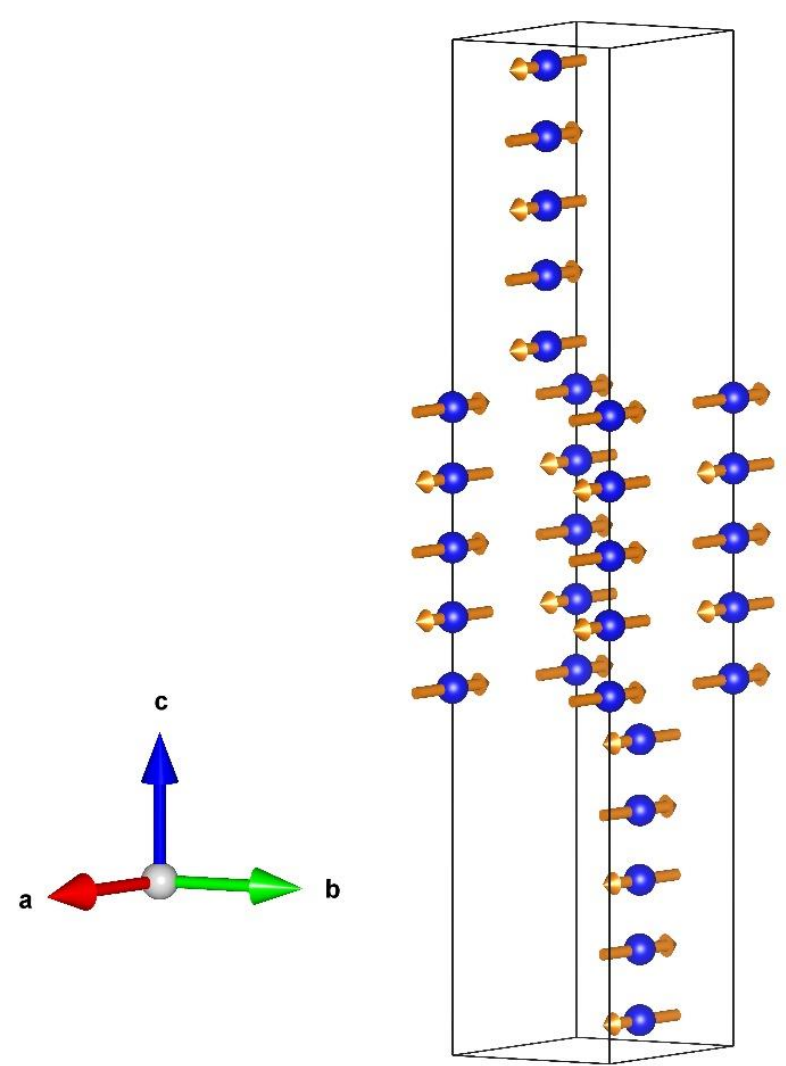

Figure S2. Spin structure in antiferromagnetic phase (below $\mathrm{T}_{\mathrm{N}} \sim 230 \mathrm{~K}$ ) for $15 \mathrm{R}-\mathrm{BaMnO}_{3}$. 

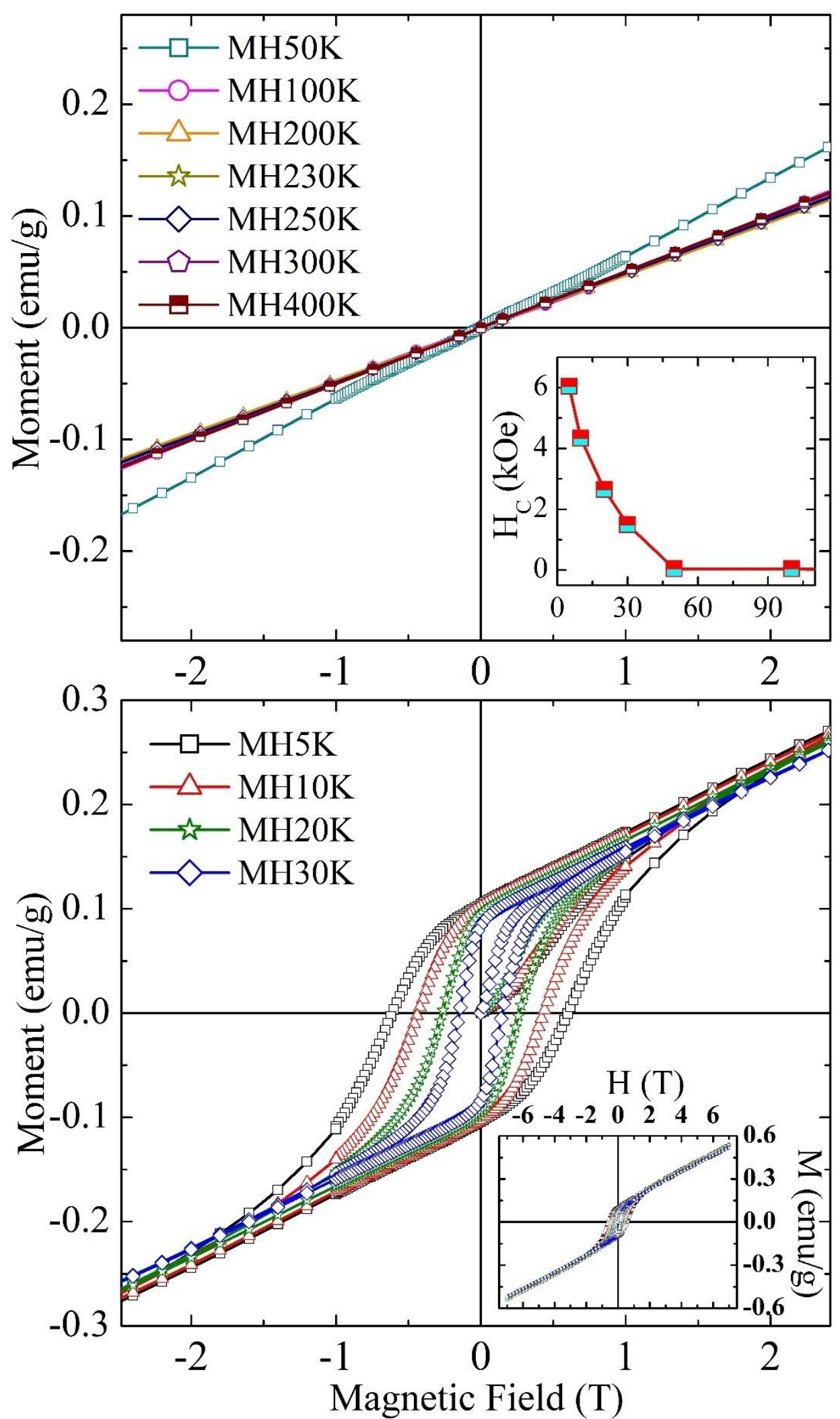

Figure S3. MH curves at a few typical temperatures. Inset in the top panel shows the plot of coercivity as a function of temperature. While the inset in the bottom panel gives the $\mathrm{M} v s \mathrm{H}$ up to the highest field $( \pm 7 \mathrm{~T})$ measured. 


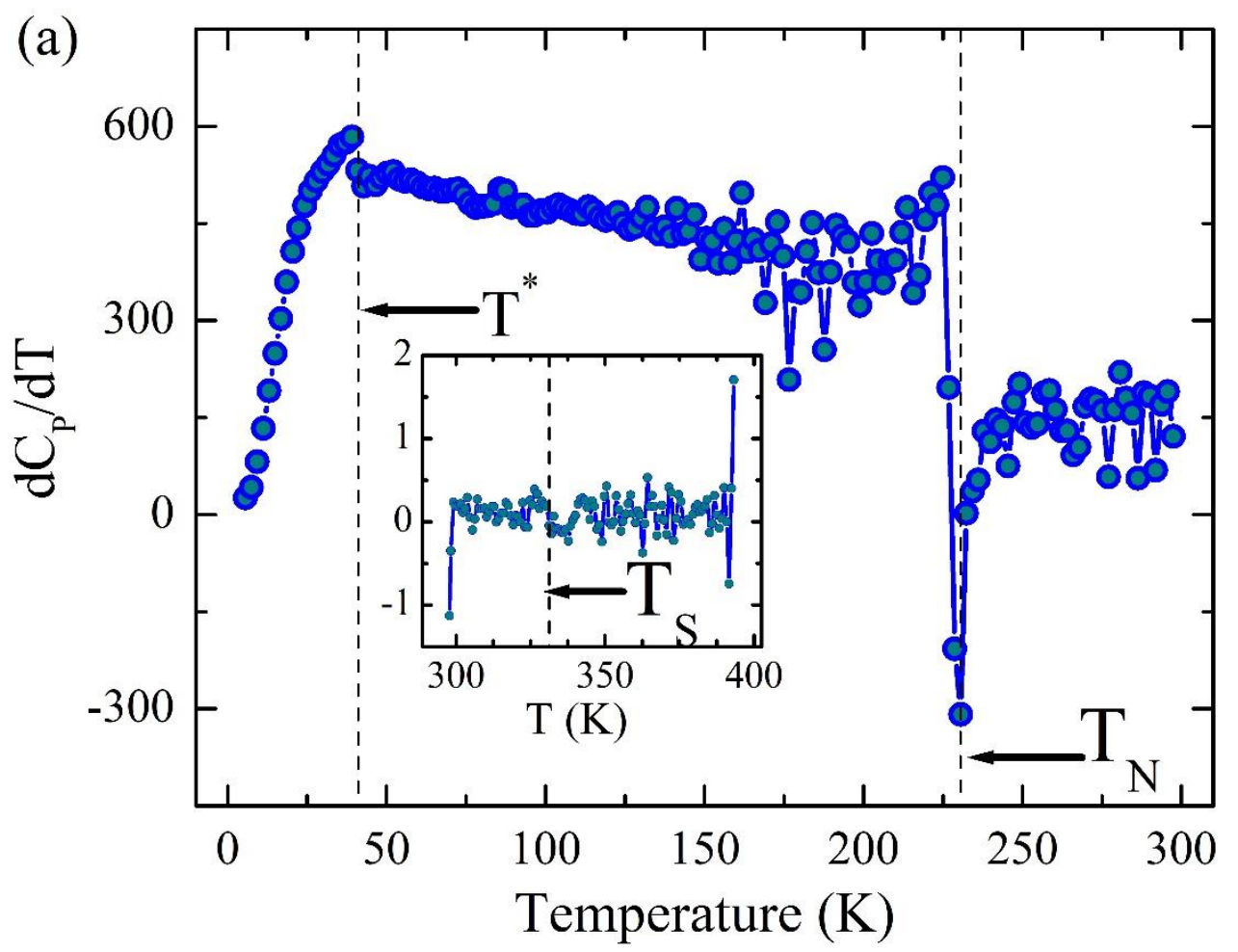

(b)

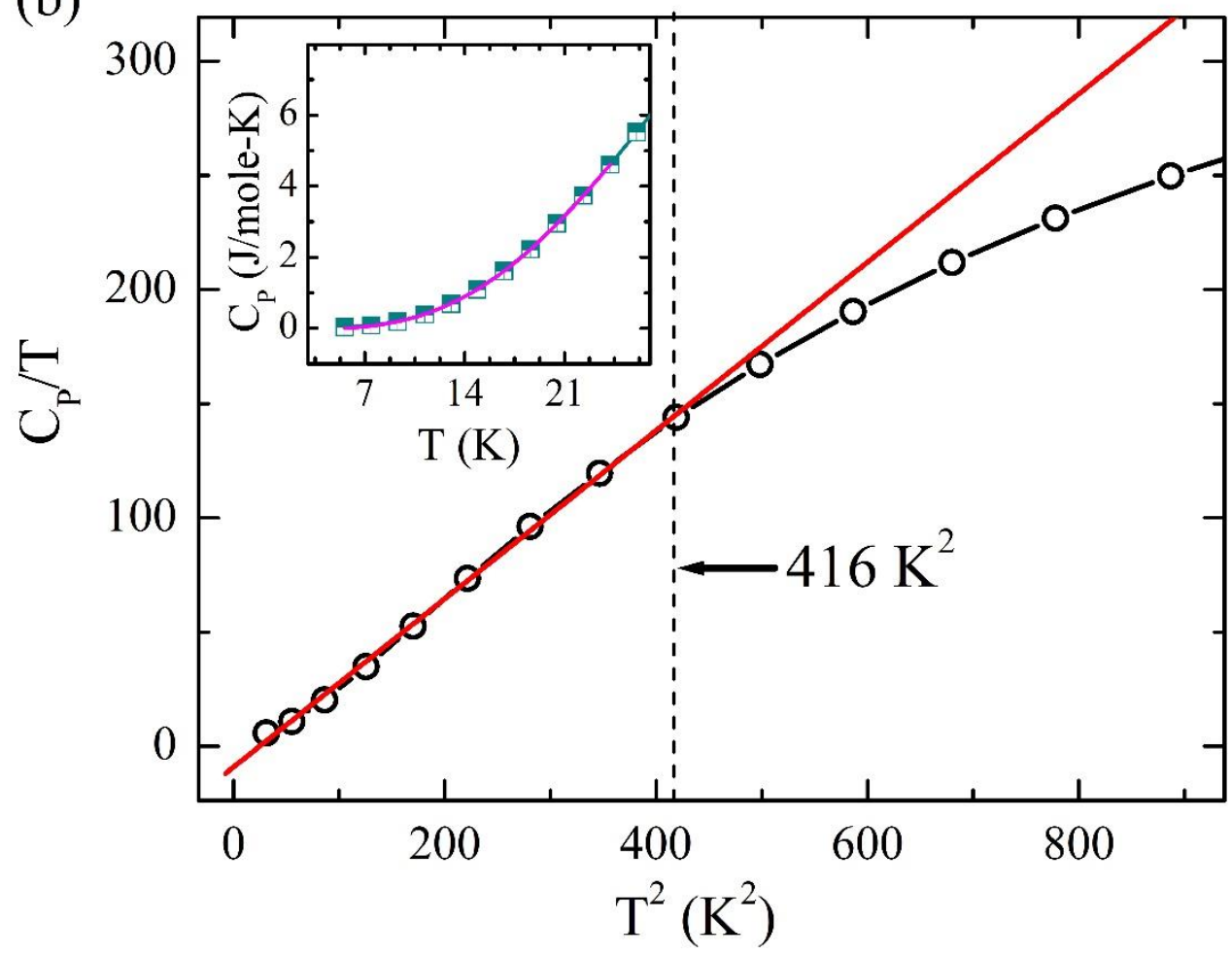

Figure S4. (a) First derivative of specific heat $\left(\mathrm{d} \mathrm{C}_{\mathrm{P}} / \mathrm{dT}\right)$ is plotted as a function of temperature, (b) $\mathrm{C}_{\mathrm{P}} / \mathrm{T}$ vs $\mathrm{T}$ plot to show the $\mathrm{T}^{3}$ dependence of specific heat in the low temperature region $(\mathrm{T}<20 \mathrm{~K})$. In the inset, the solid line is a fit with Eq. S2 explained in the text.

SM3: Symmetry assignment for Raman modes 
Raman spectra are collected in two polarization configurations as shown in Figure S5. The laser from the source is linearly polarized along $\mathrm{Y}$, the collected spectrum in the same direction of polarization is indicated as YY (parallel) polarization and collected in orthogonal direction of polarization is YX (perpendicular) polarization. The symmetry for Raman active modes is assigned based on group theoretical prediction and polarization-dependent Raman spectra. Ideally it is expected that the modes with $A_{\mathrm{g}}$ symmetry would completely get suppressed under cross polarization and $E_{\mathrm{g}}$ modes appear in both parallel and cross polarization configurations. But we have seen only slight suppression/changes in the intensity rather than mode disappearing which is due to the polycrystalline nature of the sample (where crystalline directions are not well defined) and possibly due to polarization leakage, arising from the breaking of Raman selection rules [5].

Note that the P7 mode is present in polarization-dependent Raman collected at room temperature whereas it disappears above $280 \mathrm{~K}$ in temperature-dependent measurements. It is due to the fact that the polarization measurements are performed outside the cryostat where the room temperature is close to $285 \mathrm{~K}$. According to the harmonic approximation, the phonon frequency can be expressed as $\omega \propto \sqrt{\frac{k}{m}}$. The phonon frequency is less for the vibrations involving heavier atoms and relatively high for modes associated with lighter atoms. The group theory predicts 4, 4, and 10 Raman active vibrations involving $\mathrm{Ba}, \mathrm{Mn}$, and $\mathrm{O}$ atoms, respectively, for the $15 \mathrm{R}$ phase (R-3m symmetry) as given in Table $\mathrm{S} 2$. The modes below 175 $\mathrm{cm}^{-1}$ are associated with Ba displacements. The phonons of frequency between 190 and 400 $\mathrm{cm}^{-1}$ arises from Mn vibrations. High frequency modes are related to the displacement of $\mathrm{O}$ atoms. The frequency of modes at room temperature falls at 80.9 $( \pm 0.1)\left[E_{\mathrm{g}}\right], 99.3( \pm 0.1)\left[A_{1 \mathrm{~g}}\right]$, $174.7( \pm 0.1)\left[E_{\mathrm{g}}\right], 238.9( \pm 0.1)\left[E_{\mathrm{g}}\right], 275.6( \pm 0.1)\left[E_{\mathrm{g}}\right], 358.4( \pm 0.1)\left[E_{\mathrm{g}}\right], 415.3( \pm 0.1)\left[E_{\mathrm{g}}\right]$, $427.5( \pm 0.1)\left[E_{\mathrm{g}}\right], 530.0( \pm 0.4)\left[A_{1 \mathrm{~g}}\right], 552.2( \pm 0.1)\left[E_{\mathrm{g}}\right], 578.8( \pm 0.1)\left[E_{\mathrm{g}}\right], 636.9( \pm 0.3)\left[A_{1 \mathrm{~g}}\right]$, $653.3( \pm 0.1)\left[E_{\mathrm{g}}\right] \mathrm{cm}^{-1}$. The weak modes of frequency $120.6( \pm 0.2)\left[A_{1 \mathrm{~g}}\right]$ and $195.9( \pm 0.2)$ $\left[A_{\mathrm{gg}}\right] \mathrm{cm}^{-1}$ are shown as inset of Figure S5. The symmetry of the phonon is written in square brackets. 


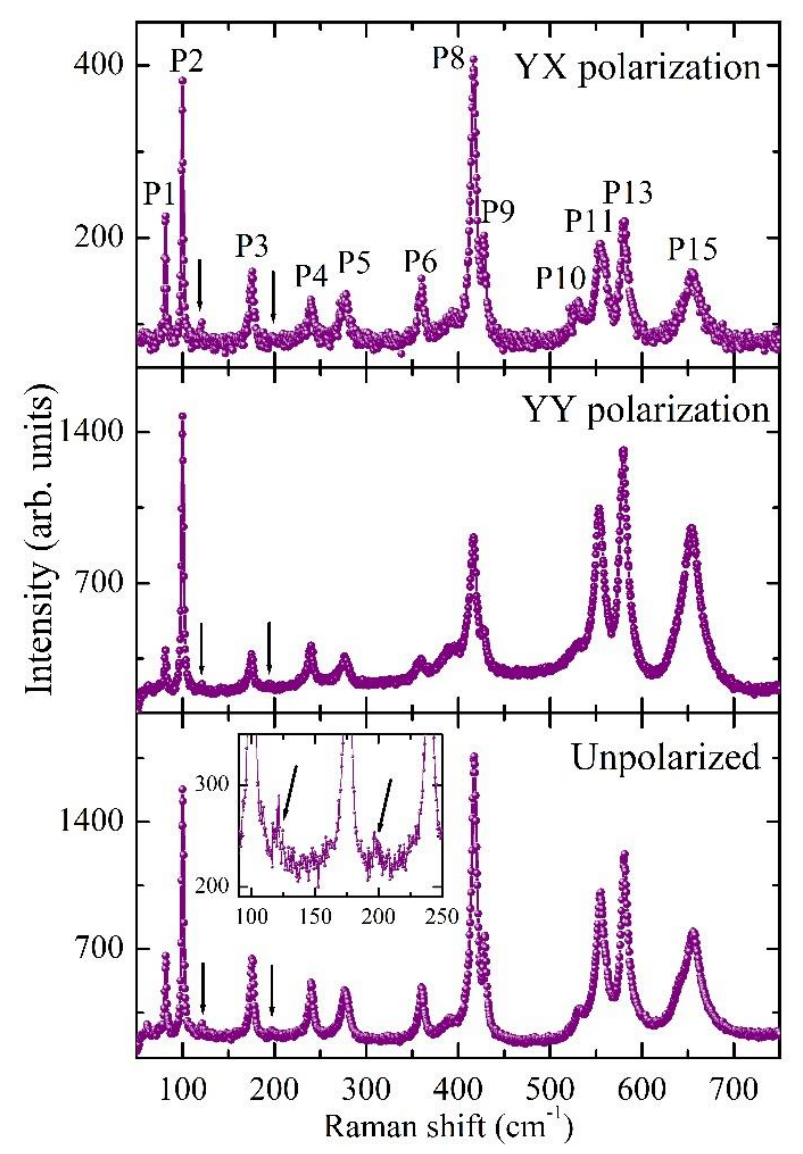

Figure S5. Raman spectra collected in parallel and perpendicular polarization configurations. Inset is enlarged view to show the weak modes at $\sim 120$ and $\sim 196 \mathrm{~cm}^{-1}$.

Table S2. Mode activity for phonons in $15 \mathrm{R}-\mathrm{BaMnO}_{3}$.

Space group: R-3m (No. 166)

It has 15 formula units (75 atoms) per unit cell

Five formula units (25 atoms) per primitive cell

\section{$\underline{\text { Wyckoff positions }}$}

Ba1: $3 b$

Ba2: $6 \mathrm{c}$

Ba3: $6 \mathrm{c}$

Mn1: 3a

Mn2: 6c

Mn3: 6c

O1: 9d $\underline{\text { Irreducible representations }}$

$$
\begin{gathered}
A_{2 u}+E_{u} \\
A_{2 u}+E_{u}+A_{1 g}+E_{g} \\
A_{2 u}+E_{u}+A_{1 g}+E_{g} \\
A_{2 u}+E_{u} \\
A_{2 u}+E_{u}+A_{1 g}+E_{g} \\
A_{2 u}+E_{u}+A_{1 g}+E_{g}
\end{gathered}
$$




\begin{tabular}{|c|c|}
\hline O2: $18 \mathrm{~h}$ & $2 A_{2 u}+3 E_{u}$ \\
O3: $18 \mathrm{~h}$ & $2 A_{2 u}+3 E_{u}+2 A_{1 g}+3 E_{g}$ \\
& $2 A_{2 u}+3 E_{u}+2 A_{1 g}+3 E_{g}$ \\
\hline Total irreducible representations $\Gamma_{\text {Raman }}=8 A_{1 g}+10 E_{g}$ and $\Gamma_{I R}=12 A_{2 u}+15 E_{u}$ \\
\hline
\end{tabular}

\section{SM4: Temperature-dependent Raman spectra}

The Raman spectra at a few typical temperatures are stacked in Figure S6. The fitting parameters extracted from cubic anharmonic equations for temperature-dependent frequency and linewidth are listed in Table S3. Phonon parameters (intensity, frequency, and linewidth) for P1-P5, P9, P11, and P13 modes are plotted as a function of temperature in Figure S7-S9. The finite deviation from the expected anharmonic behaviour is observed for all modes which can be attributed to magnetostriction in the system.

Table S3. Anharmonic fitting (Eq. 2 and 3 in main text) parameters for Raman active phonons in $15 \mathrm{R}-\mathrm{BaMnO}_{3}$.

\begin{tabular}{|c|c|c|c|c|c|}
\hline Mode & $\begin{array}{l}\text { Temperature } \\
\text { range where } \\
\text { the mode } \\
\text { exists }\end{array}$ & $\omega_{0}\left(\mathrm{~cm}^{-1}\right)$ & $\mathbf{A}$ & $\Gamma_{0}\left(\mathrm{~cm}^{-1}\right)$ & C \\
\hline $\mathrm{P} 1$ & $80-800 \mathrm{~K}$ & $82.42 \pm 0.06$ & $-0.030 \pm 0.001$ & $1.30 \pm 0.06$ & $\begin{array}{c}0.020 \pm \\
0.001\end{array}$ \\
\hline $\mathrm{P} 2$ & $80-800 \mathrm{~K}$ & $100.90 \pm 0.06$ & $-0.030 \pm 0.001$ & $1.50 \pm 0.06$ & $\begin{array}{c}0.020 \pm \\
0.001\end{array}$ \\
\hline $\mathrm{P} 3$ & $80-800 \mathrm{~K}$ & $177.50 \pm 0.09$ & $-0.100 \pm 0.002$ & $1.80 \pm 0.15$ & $\begin{array}{c}0.130 \pm \\
0.003\end{array}$ \\
\hline $\mathrm{P} 4$ & $80-800 \mathrm{~K}$ & $242.10 \pm 0.09$ & $-0.150 \pm 0.002$ & $2.10 \pm 0.24$ & $0.22 \pm 0.01$ \\
\hline P5 & $80-800 \mathrm{~K}$ & $279.60 \pm 0.10$ & $-0.240 \pm 0.003$ & $1.30 \pm 0.28$ & $0.50 \pm 0.01$ \\
\hline P6 & $240-800 \mathrm{~K}$ & $365.70 \pm 0.24$ & $-0.540 \pm 0.009$ & $1.60 \pm 0.29$ & $0.50 \pm 0.01$ \\
\hline P7 & $80-280 \mathrm{~K}$ & \multicolumn{4}{|c|}{$\begin{array}{l}\text { Anharmonic equation is not fitted as this mode is no longer } \\
\text { purely anharmonic }\end{array}$} \\
\hline P8 & $240-800 \mathrm{~K}$ & $421.80 \pm 0.33$ & $-0.560 \pm 0.016$ & $0.01 \pm 0.00$ & $1.00 \pm 0.02$ \\
\hline P9 & $80-800 \mathrm{~K}$ & $431.38 \pm 0.11$ & $-0.330 \pm 0.006$ & $0.84 \pm 0.15$ & $0.32 \pm 0.01$ \\
\hline
\end{tabular}




\begin{tabular}{|c|c|c|c|c|c|}
\hline P10 & $80-800 \mathrm{~K}$ & $536.54 \pm 0.55$ & $-0.510 \pm 0.035$ & $8.38 \pm 2.09$ & $1.67 \pm 0.13$ \\
\hline P11 & $80-800 \mathrm{~K}$ & $561.10 \pm 0.22$ & $-1.030 \pm 0.015$ & $3.33 \pm 0.38$ & $0.89 \pm 0.02$ \\
\hline P12 & $80-220 \mathrm{~K}$ & \multicolumn{4}{|c|}{$\begin{array}{l}\text { Anharmonic equation is not fitted as this mode is no longer } \\
\text { purely anharmonic }\end{array}$} \\
\hline P13 & $80-800 \mathrm{~K}$ & $584.71 \pm 0.13$ & $-0.690 \pm 0.009$ & $2.12 \pm 0.41$ & $0.95 \pm 0.03$ \\
\hline P14 & $80-330 \mathrm{~K}$ & \multicolumn{4}{|c|}{$\begin{array}{l}\text { Anharmonic equation is not fitted as this mode is no longer } \\
\text { purely anharmonic }\end{array}$} \\
\hline P15 & $240-800 \mathrm{~K}$ & $658.68 \pm 0.24$ & $-0.710 \pm 0.017$ & $13.10 \pm 0.55$ & $1.56 \pm 0.04$ \\
\hline
\end{tabular}
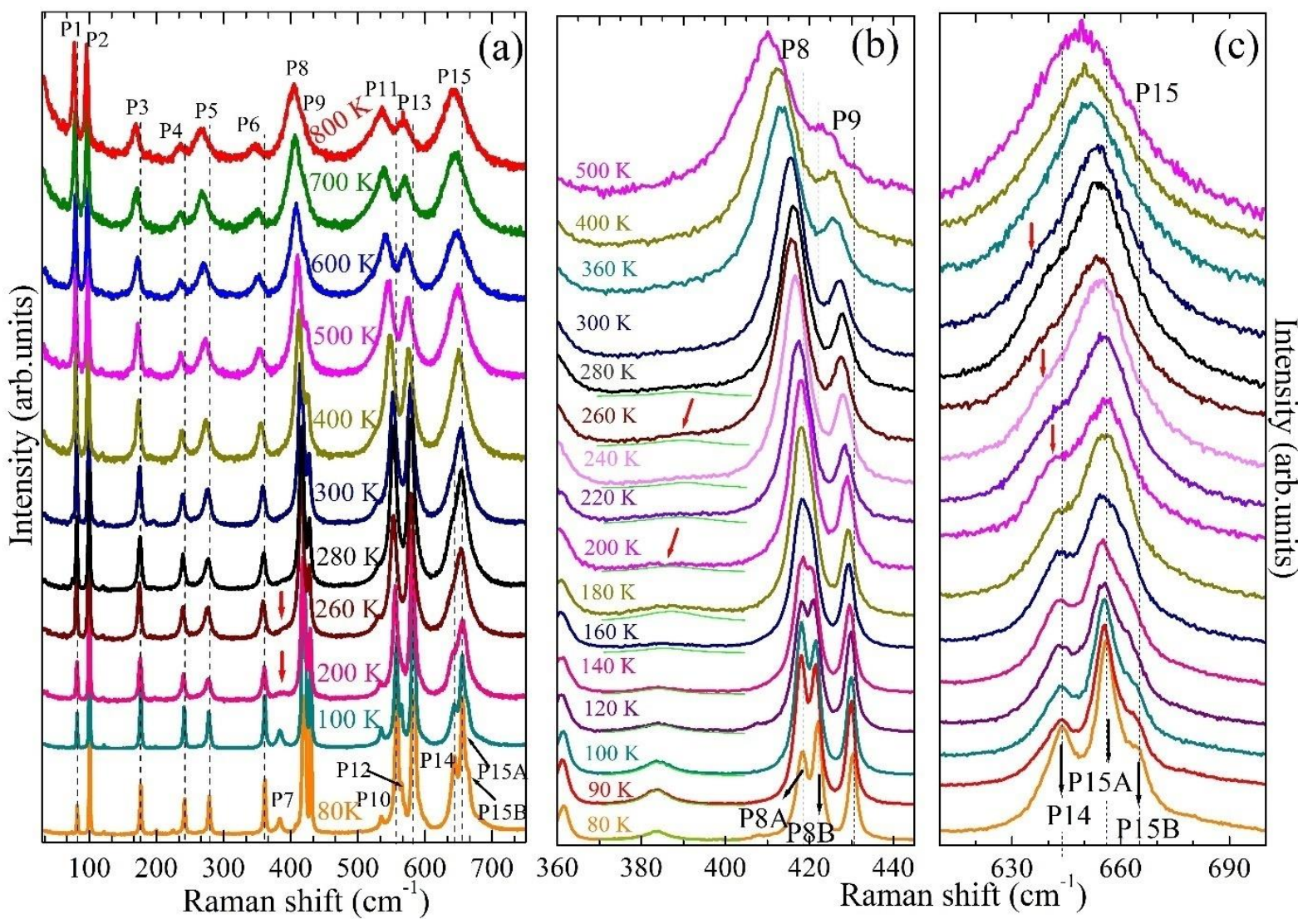

Figure S6. (a) Raman spectra of $15 \mathrm{R}-\mathrm{BaMnO}_{3}$ collected at a few temperatures where $\mathrm{P} 7$ disappears above $\sim 280 \mathrm{~K}\left(\mathrm{~T}_{\mathrm{D}}\right)$, (b) and (c) show the enlarged view to show the splitting of P8 and P15 phonons, respectively below magnetic ordering temperature $\left(\mathrm{T}_{\mathrm{N}}\right)$. 


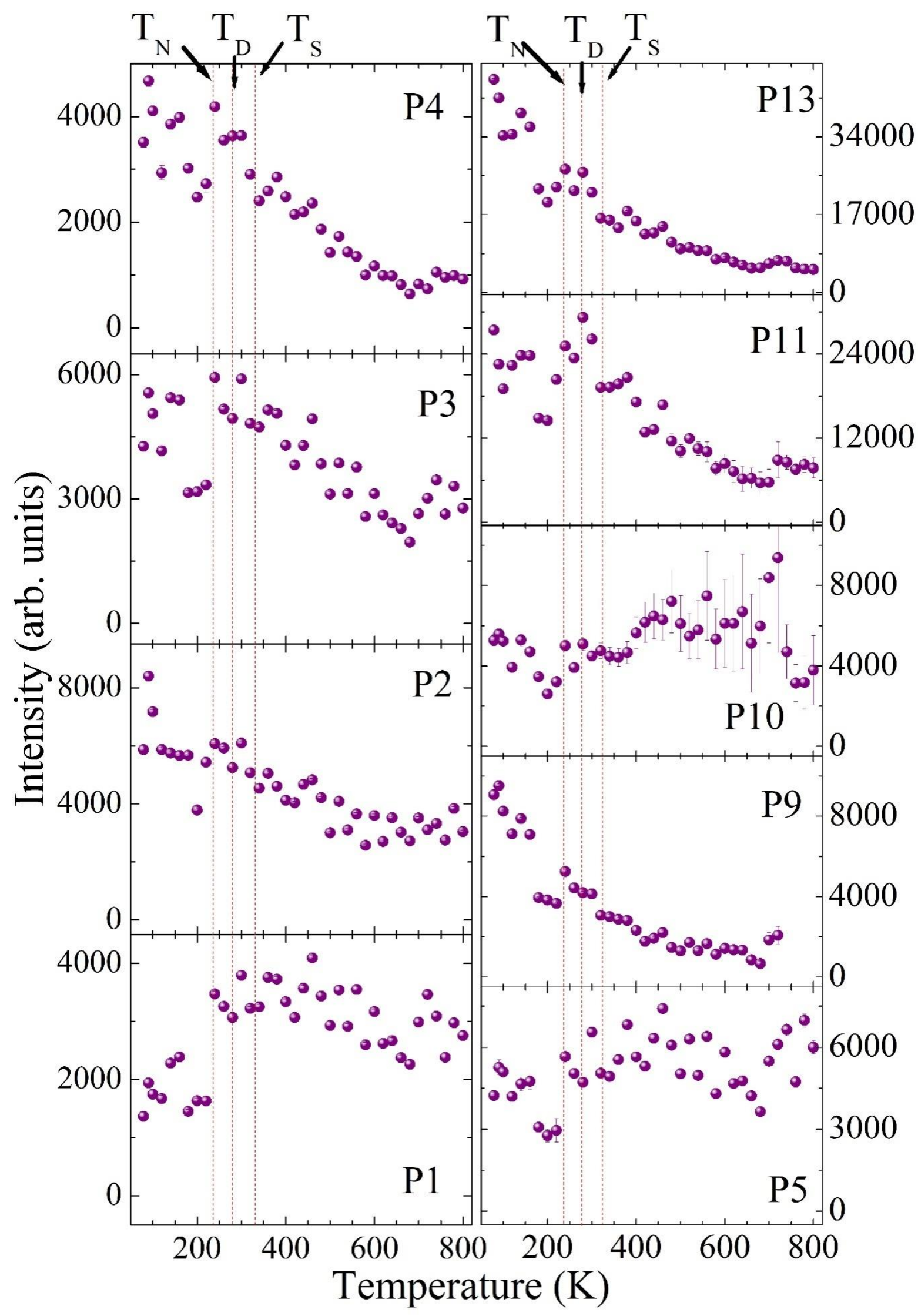

Figure S7. Spectral weight of a few Raman active phonons as a function of temperature. 


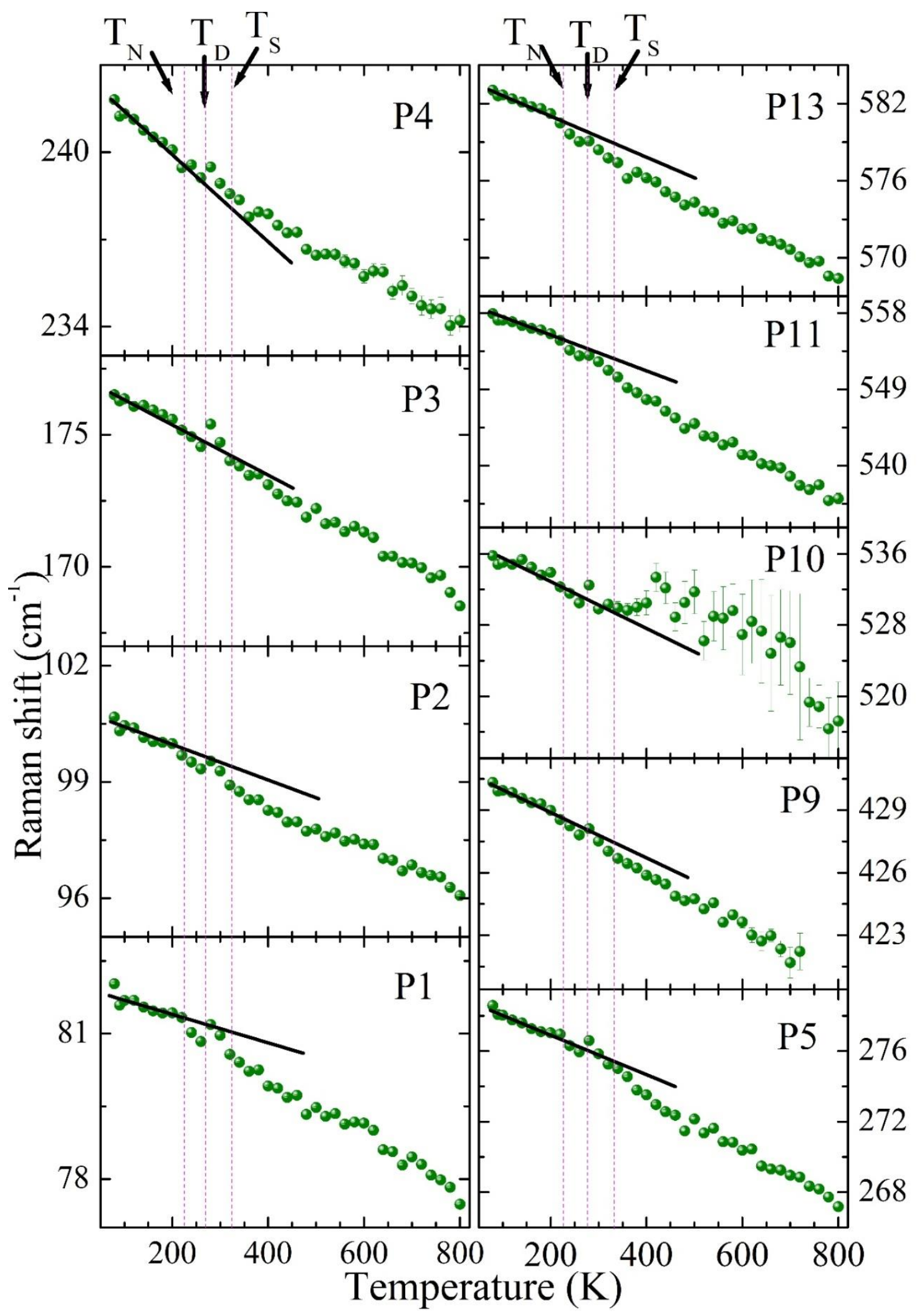

Figure S8. Frequency of a few Raman active phonons as a function of temperature. Solid lines are guide to eye showing the deviation at magnetic transition temperatures. 


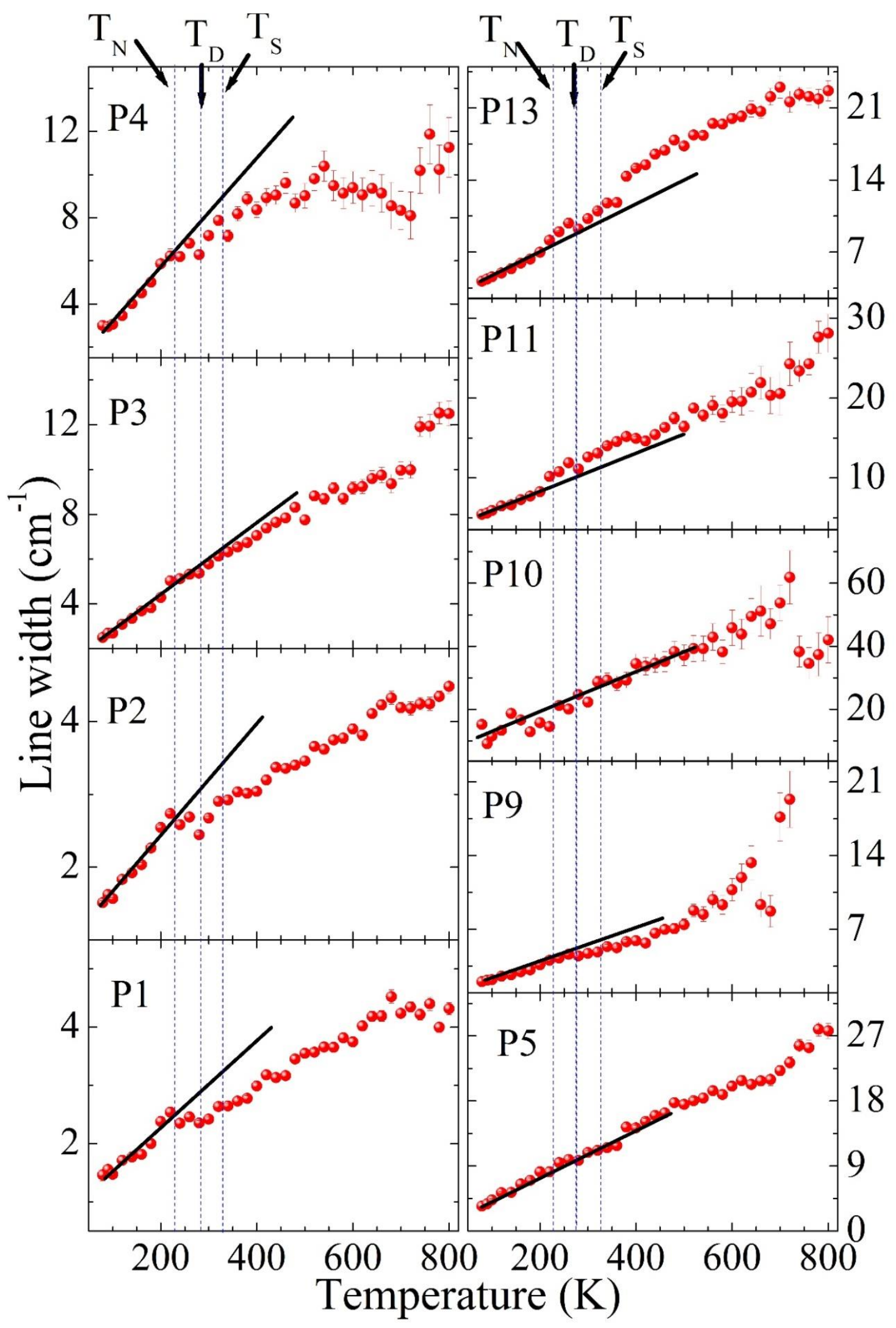

Figure S9. Linewidth of a few Raman active phonons as a function of temperature. Solid lines are guide to eye showing the deviation at magnetic transition temperatures. 


\section{SM5: Spin-phonon coupling}

We have analysed the temperature-dependent phonon frequency using Eq. 2 (explained in main text). The deviation from the anharmonic equation (Eq. 2 ) is calculated by the equation:

$$
\Delta \omega(T)=\omega(T)-\omega_{\text {anh }}(T)
$$

In magnetic materials, the phonon parameters such as frequency and linewidth may respond to the magnetic transition temperature $\left(\mathrm{T}_{\mathrm{N}}\right)$ due to spin-phonon coupling. The change in phonon frequency due to spin-phonon coupling $(\lambda)$ in magnetically ordered phase can be written as:

$$
\omega(T)=\omega_{\text {anh }}(T)-\lambda<S_{i} . S_{j}>
$$

where $<S_{i} . S_{j}>$ defines the spin-spin correlation. The deviation in the phonon frequency from the expected anharmonic behaviour in the magnetically ordered phase is:

$$
\Delta \omega(T)=\Delta \omega_{s p-p h}(T)=\omega(T)-\omega_{a n h}(T)=-\lambda<S_{i} . S_{j}>
$$

We have estimated the spin-phonon coupling constant using the following relation as explained in the main text:

$$
\lambda_{s p}=-\frac{\omega\left(T_{\text {Low }}\right)-\omega_{\text {anh }}\left(T_{\text {Low }}\right)}{\left[\Phi\left(T_{\text {Low }}\right)-\Phi\left(2 T_{N}\right)\right] S^{2}}
$$

where $\omega\left(T_{\text {Low }}\right)$ is the experimental phonon frequency at the lowest temperature $(\mathrm{T} \sim 80 \mathrm{~K})$ recorded while the $\omega_{\text {anh }}\left(T_{\text {Low }}\right)$ is the anharmonic estimate of the phonon frequency at the same temperature. The spin (S) is $3 / 2$ for $\mathrm{Mn}^{+4} . \Phi$ is the short-range order parameter. Since P7 phonon does not exist in paramagnetic phase, in the absence of experimental data with anharmonic behaviour, we have used $\omega$ at $\mathrm{T}_{\mathrm{N}}\left(\omega_{T_{N}}\right)$ in the above equation for $\omega_{\text {anh }}\left(T_{\text {Low }}\right)$, to estimate $\lambda_{s p}$. This procedure will result in underestimation of the $\lambda_{s p}$ for P7.

(a)

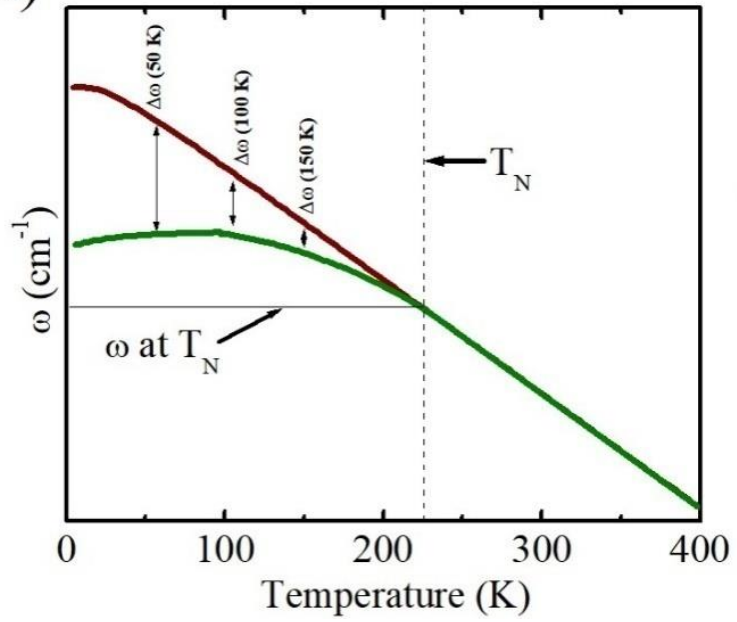

(b)

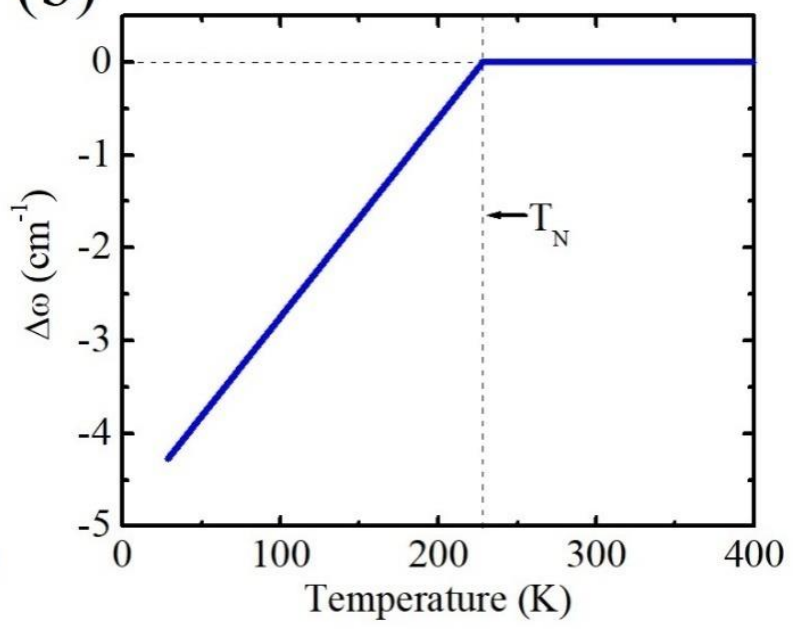

Figure S10. The schematics for (a) $\omega v s T$ showing the deviation from the expected anharmonic trend, and (b) $\Delta \omega_{s p-p h}(T)$ vs $T$. 


\section{SM6: Magnetic field dependent Raman spectra}

Figure S11a shows the Raman spectra collected at a few magnetic fields. The phonon intensity and linewidth for spin-related modes as a function of temperature are plotted in Figure S11b. Intensity, frequency, and linewidth for other modes are plotted in Figures S12, S13, and S14, respectively.
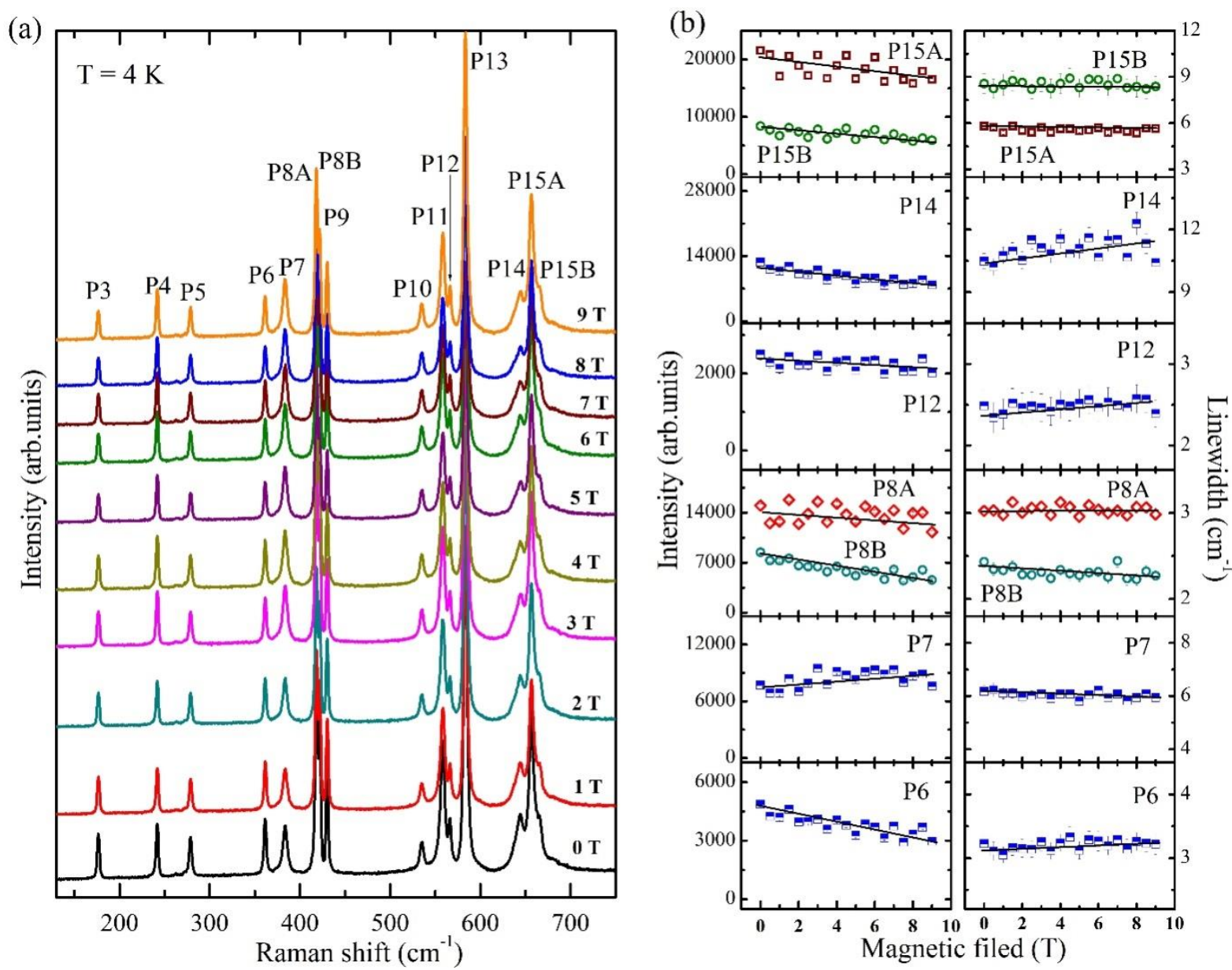

Figure $\mathrm{S} 11$. (a) Raman spectra of $15 \mathrm{R}-\mathrm{BaMnO}_{3}$ collected at a few magnetic fields, (b) intensity and linewidth of spin-related modes as a function of magnetic field. 


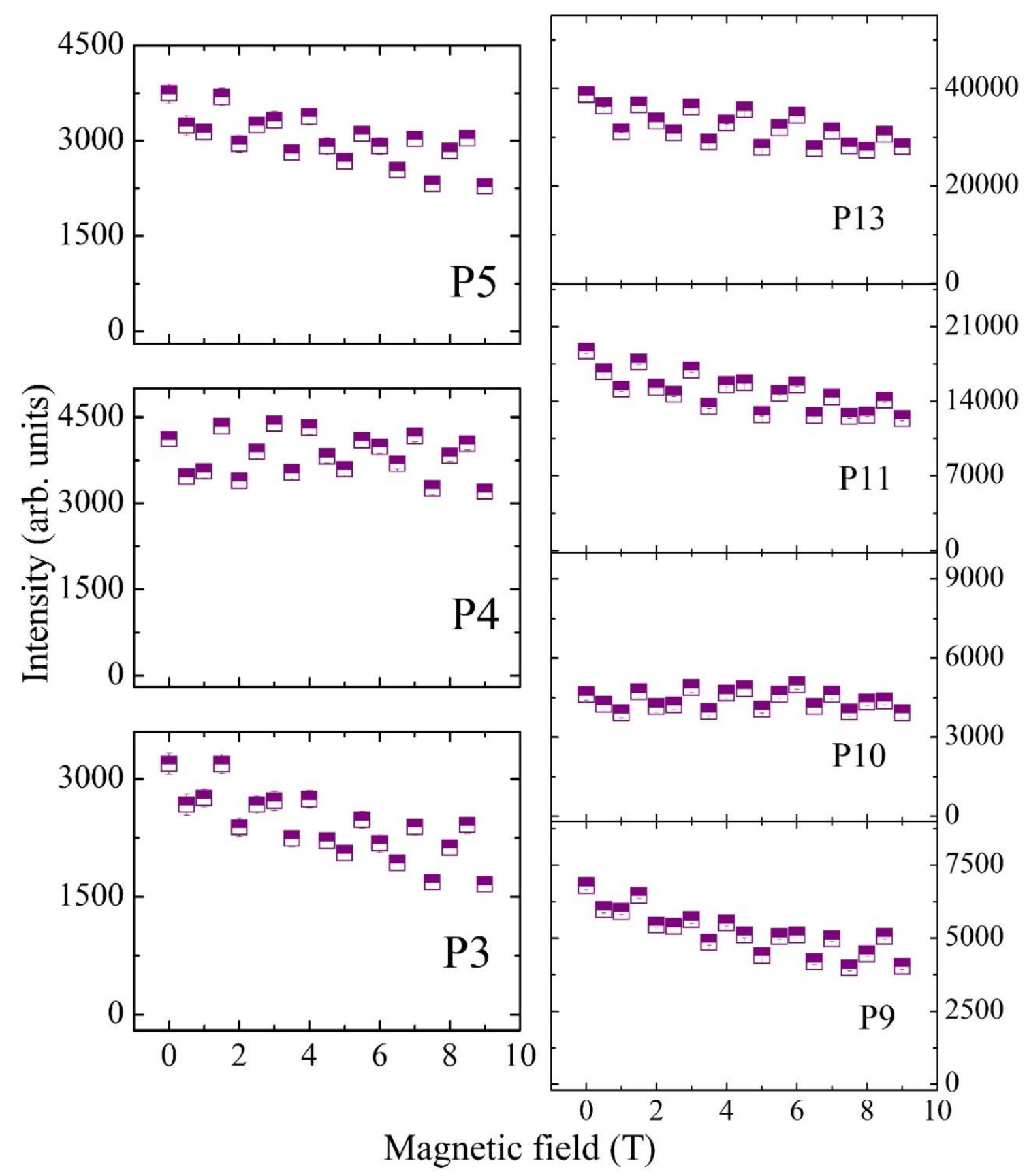

Figure S12. Spectral weight of phonons as a function of applied magnetic field 


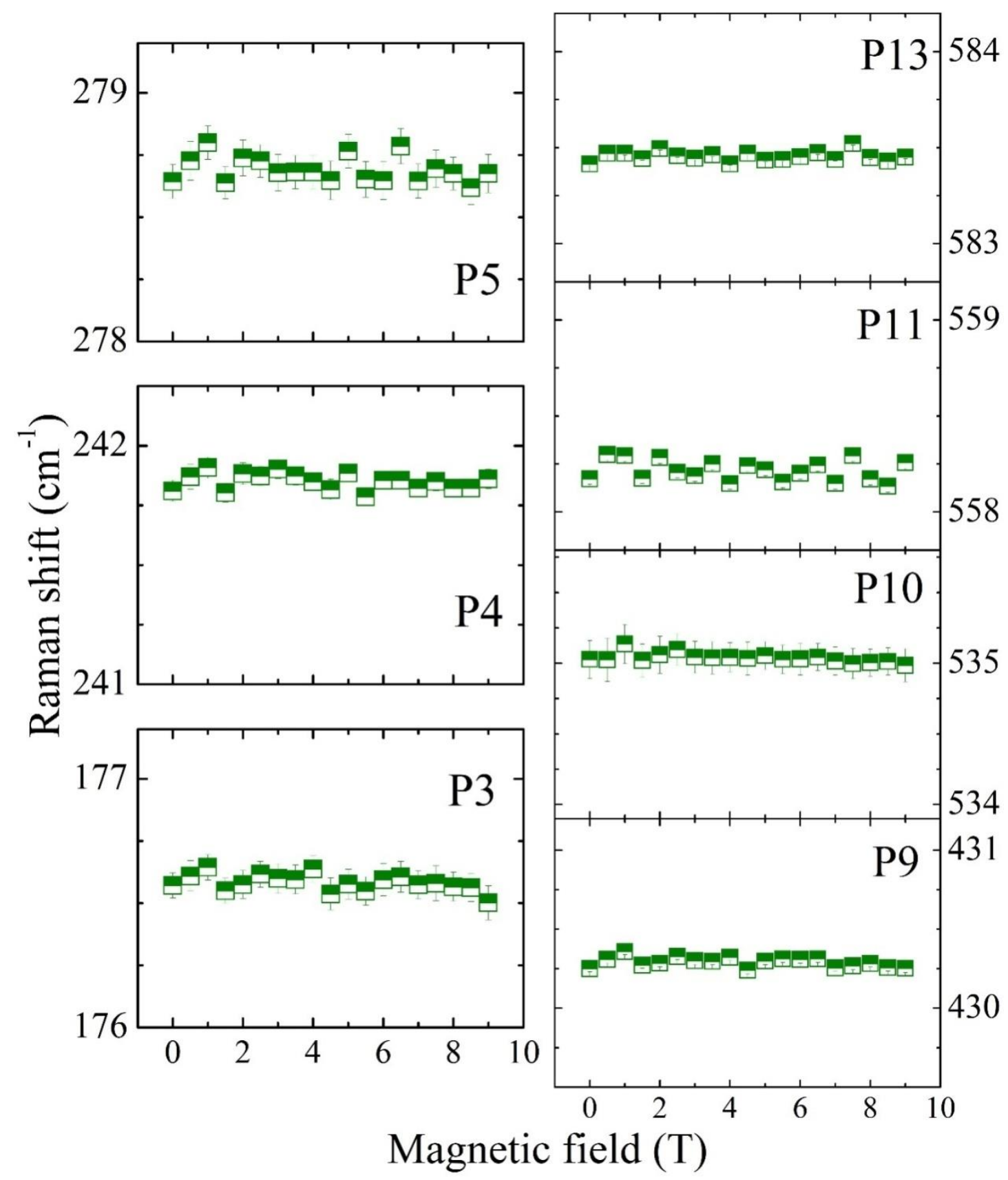

Figure S13. Frequency of phonon as a function of applied magnetic field 


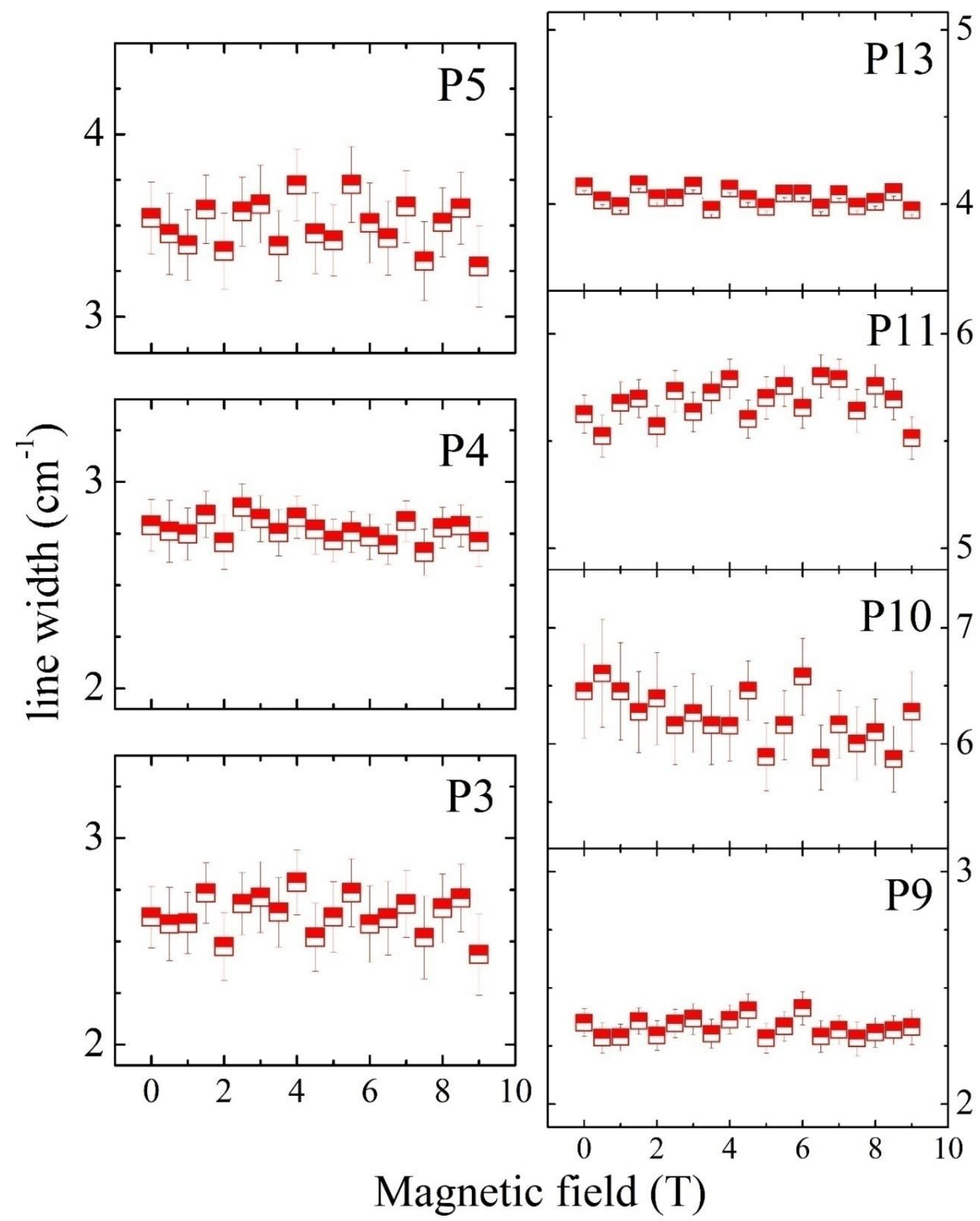

Figure S14. Phonon linewidth as a function of applied magnetic field

\section{SM7: Thermal expansion: Temperature-dependent $x$-ray diffraction}

The x-ray diffraction patterns collected at a few typical temperatures (Figure 6a in main text) confirms that the crystal symmetry remains the same throughout the investigated temperature range (90-450 K). The temperature-dependent lattice parameters due to thermal expansion can be written as [6]: 


$$
a(T)=a_{0}\left[1+\frac{b e^{\frac{d}{T}}}{T\left(e^{\frac{d}{T}}-1\right)^{2}}\right] \quad \text { and } \quad c(T)=c_{0}\left[1+\frac{f e^{\frac{g}{T}}}{T\left(e^{\frac{g}{T}}-1\right)^{2}}\right]
$$

where, $a_{0}$ and $\mathrm{c}_{0}$ are the in-plane and out-of-plane lattice constants at $0 \mathrm{~K}$, whereas $\mathrm{b}, \mathrm{d}, \mathrm{f}$, and $\mathrm{g}$ are fitting parameters. Unit cell volume expansion with temperature can be expressed as:

$$
V(T)=V_{0}\left[1+\frac{A}{\left(e^{\frac{\theta}{T}}-1\right)}\right]
$$

where, $V_{0}$ is the cell volume extrapolated to $0 \mathrm{~K}$ and $\theta$ is Debye temperature and $\mathrm{A}$ is fitting constant. The fitting parameters are given in table S4. The lattice parameters as a function of temperature are shown in Figure $6 \mathrm{~b}$ in main text. The fitted data in the entire temperature range (90-450 K) does not give reliable values for parameters. The best fitted parameters of temperature-dependent lattice constants with Eq. S3 and S4 are given in Table S3. The fluctuations in lattice parameters around $\mathrm{T}_{\mathrm{S}}$ and $\mathrm{T}_{\mathrm{D}}$ represent the local modifications in the lattice due to magnetic ordering and the change in electric property. However, unit cell volume fits reasonably well with Eq. (5) in the entire temperature range (90-450 K). The Debye temperature from x-ray diffraction results is $\theta \sim 352 \pm 20 \mathrm{~K}$. Figure S15 shows the Mn-Mn separation as a function of temperature. All the Mn-Mn bond lengths show an increasing trend with increasing temperature and exhibit a change in slope at $\mathrm{T}_{\mathrm{S}}$, similar to the lattice parameters.

Table S4. The best fit parameters of temperature-dependent lattice parameters with Eq. 4 and 5 explained in the main text.

\begin{tabular}{|c|c|c|}
\hline $\begin{array}{c}\text { Lattice } \\
\text { parameter }(\AA)\end{array}$ & $\begin{array}{c}\text { Selected } \\
\text { temperature } \\
\text { range }(\mathrm{K})\end{array}$ & Fitting parameters \\
\hline $\mathrm{a}$ & $90-450 \mathrm{~K}$ & $\begin{array}{r}a_{0}=5.6686 \pm 0.0028 \AA, \mathrm{b}=0.8607 \pm 1.2737, \\
\text { and } \mathrm{d}=327.12 \pm 255.587\end{array}$ \\
\hline $\mathrm{c}$ & $90-330 \mathrm{~K}$ & $\begin{array}{r}a_{0}=5.6692 \pm 0.0005 \AA, \mathrm{b}=1.8253 \pm 0.5526, \\
\text { and } \mathrm{d}=454.14 \pm 64.602\end{array}$ \\
\hline $\mathrm{V}$ & $90-450 \mathrm{~K}$ & $\begin{array}{r}c_{0}=35.293 \pm 0.0171 \AA, \mathrm{f}=0.8502 \pm 1.3009, \\
\text { and } \mathrm{g}=336.16 \pm 271.17\end{array}$ \\
\hline & $90-330 \mathrm{~K}$ & $\begin{array}{r}c_{0}=35.297 \pm 0.0028 \AA, \mathrm{f}=2.0171 \pm 0.5038, \\
\text { and } \mathrm{g}=488.93 \pm 57.128\end{array}$ \\
\hline & $90-450 \mathrm{~K}$ & $\begin{array}{r}V_{0}=1134 \pm 0.25 \AA^{3}, \mathrm{~A}=23.76 \pm 0.86 \text { and } \\
\theta \sim 352 \pm 20.33 \mathrm{~K} .\end{array}$ \\
\hline & $\begin{array}{r}V_{0}=1134 \pm 0.23 \AA^{3}, \mathrm{~A}=27.33 \pm 2.22 \text { and } \\
\theta\end{array}$ \\
\hline
\end{tabular}



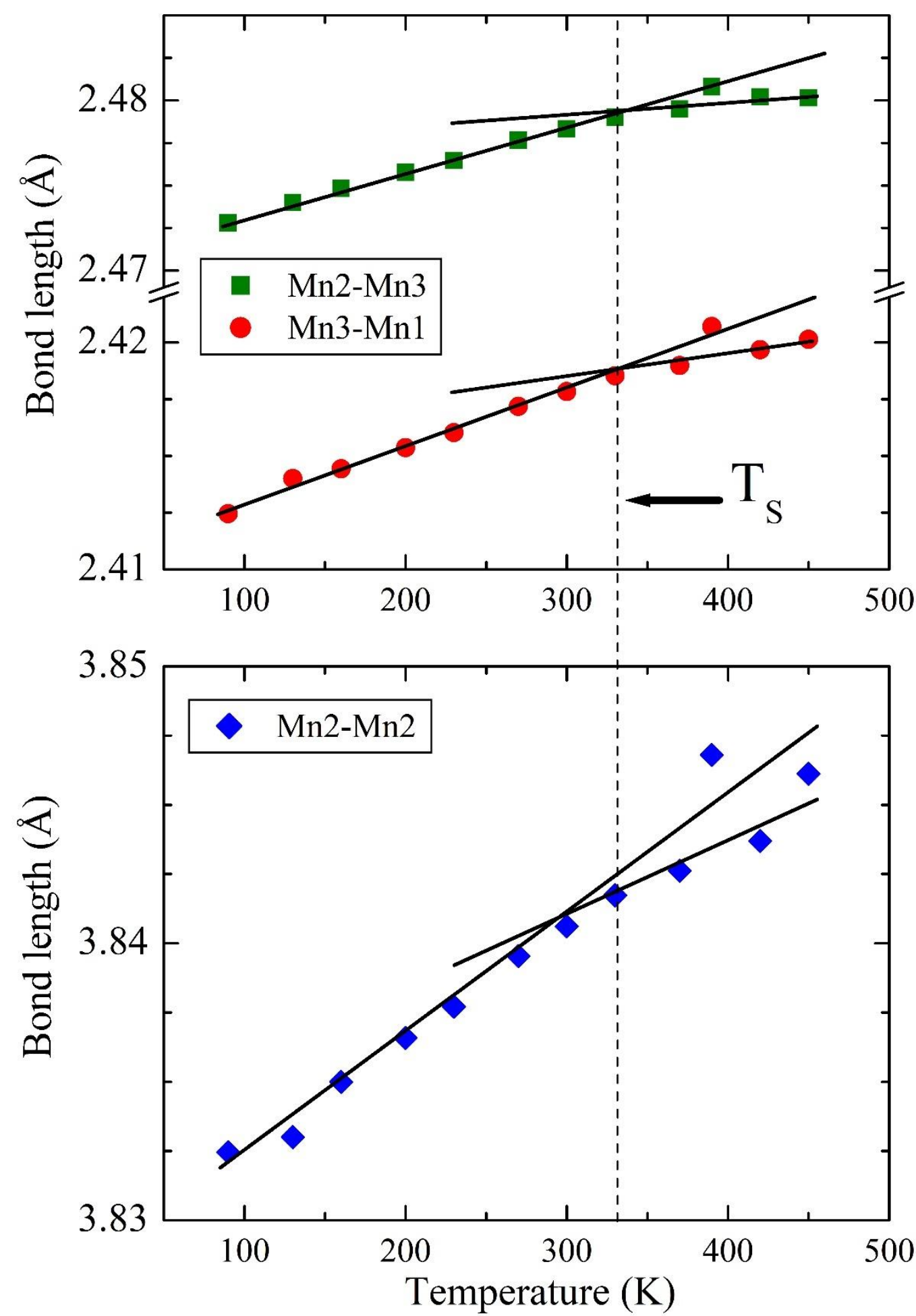

Figure S15: Mn-Mn separation as a function of temperature. Solid lines are guide to eye showing the deviation at $\mathrm{T}_{\mathrm{S}}$. 


\section{SM8: Magnetostriction}

\section{Magnetostriction from $x$-ray diffraction measurements}

The spontaneous volume magnetostriction $\left(\lambda_{m s}^{V}\right)$ at a given temperature can be defined as the difference between the volume of the unit cell $\left(V_{A F M}(T)\right)$ in the magnetically ordered phase (antiferromagnet in $15 \mathrm{R}-\mathrm{BaMnO}_{3}$ ) at that temperature and the hypothetical volume of the unit cell at the same temperature if it were in the paramagnetic (nonmagnetic) phase $\left(V_{P M}(T)\right.$ ) normalized with respect to the paramagnetic unit cell volume $\left(V_{P M}(T)\right.$ ). It can be expressed (as shown by equation-8 in main text) as $\lambda_{m s}^{V}(T)=\frac{V_{A F M}(T)-V_{P M}(T)}{V_{P M}(T)}$. The $\lambda_{m s}^{V}$ estimated from powder x-ray diffraction measurements (PXRD) as a function of temperature is shown in Figure S16.

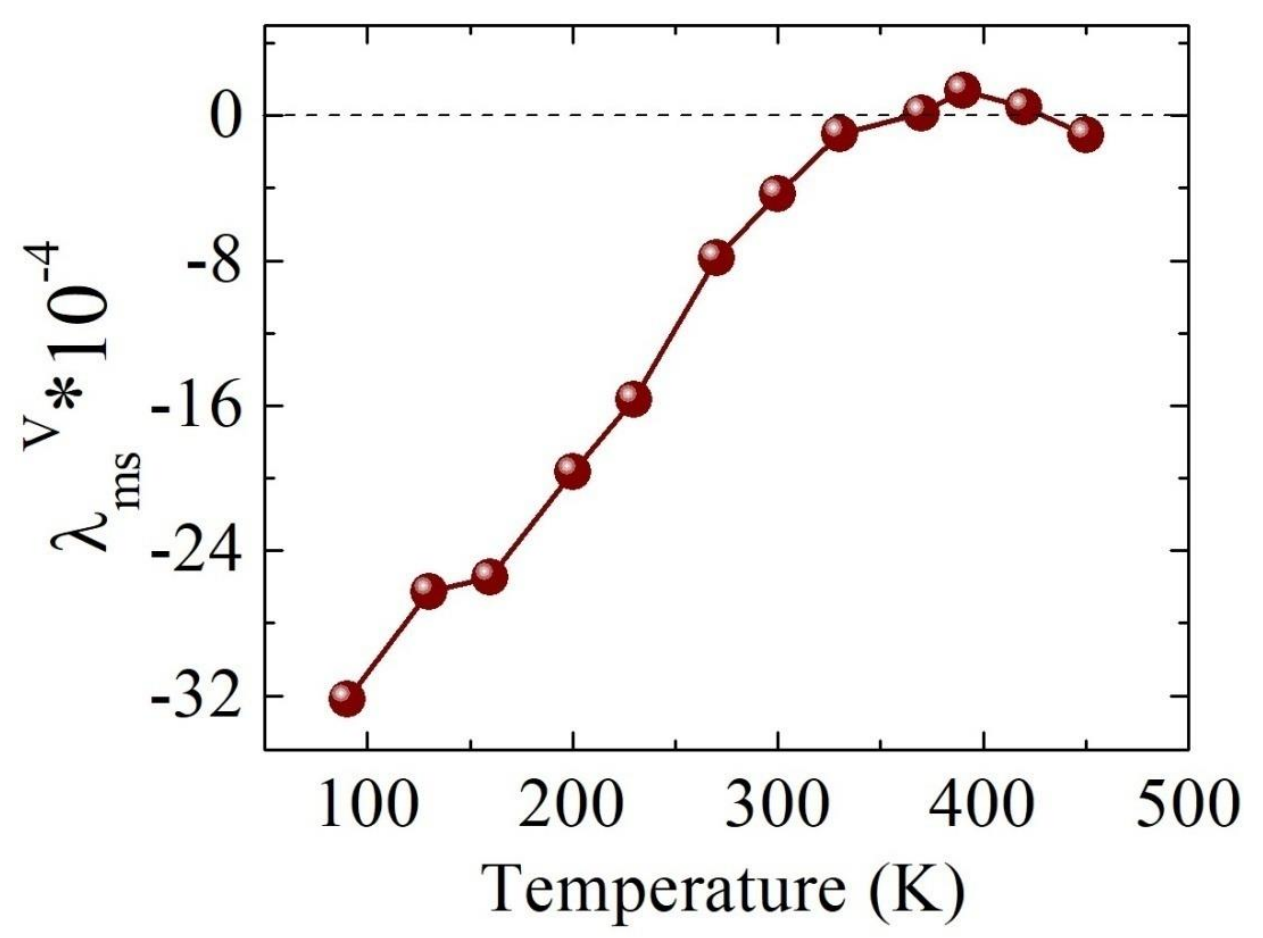

Figure S16. The spontaneous volume magnetostriction $\left(\lambda_{m s}^{V}\right)$ of $15 \mathrm{R}-\mathrm{BaMnO}_{3}$ as a function of temperature (estimated from PXRD).

\section{Estimation of Grüneisen parameter}

We have calculated $\frac{\Delta \omega}{\omega}=\frac{\omega_{T}-\omega_{80 K}}{\omega_{80 K}}$ and $\frac{\Delta V}{V}=\frac{V_{T}-V_{80 K}}{V_{80 K}}$ from temperature-dependent Raman and XRD results, respectively (Figures S17 and S18). Considering the relation $\frac{\Delta \omega}{\omega}(T)=$ $-\gamma \frac{\Delta V}{V}(T)$, we have estimated the Grüneisen parameter $(\gamma)$ for all the modes which show linear relation between $\frac{\Delta \omega}{\omega}(T)$ and $\frac{\Delta V}{V}(T)$ in the temperature range of $80-330 \mathrm{~K}$ as shown in Figure S19 and the value is found to be in between 1 and 2 (displayed in Figure S19). 


\section{Magnetostriction from Raman measurements}

The magnetostriction is defined as the change in the physical dimension caused by magnetization from the demagnetized state to magnetic saturation. Magnetostriction constant is represented by $\frac{\Delta l}{l}$, the change in the length by original length at zero field. Here, we have measured temperature-dependent magnetization under different magnetic fields in the range of 1-7T. The bifurcation between ZFC and FC curves is observed to decrease with applied magnetic field and then completely merges at 7T (Figure S20) which means that the antiferromagnetic state switches to ferromagnetic state at higher magnetic fields. We have collected Raman spectra in this range (1-9T) and estimated the change in phonon frequency due to the magnetic field at a fixed temperature $(T=4 \mathrm{~K})$ using $\frac{\Delta \omega}{\omega}=\frac{\omega_{H}-\omega_{0 T}}{\omega_{0 T}}$. The change in the phonon frequency associates with a change in volume due to applied magnetic field through the respective Grüneisen parameter $(\gamma): \frac{\Delta \omega}{\omega}(H)=-\gamma \frac{\Delta V}{V}(H)$. Under the assumption of $\gamma \sim 1$, $\frac{\Delta V}{V}(H)=-\frac{\Delta \omega}{\omega}(H)$, therefore, the volume magnetostriction is $\lambda_{V}=\frac{\Delta V}{V}(H) \approx-\frac{\Delta \omega}{\omega}(H)$. The value of $\lambda_{V}$ for the different phonons is found to be in the range of $3 \times 10^{-4}$ to $14 \times 10^{-4}$ (Figure $\mathrm{S} 21)$. The values of $\lambda_{V}$ found for $15 \mathrm{R}-\mathrm{BaMnO}_{3}$ obtained from our results are comparable to several reported values for polycrystalline materials as listed in Table S5 [7-14]. Further, the values of magnetostriction obtained from x-ray and Raman measurements are comparable. 

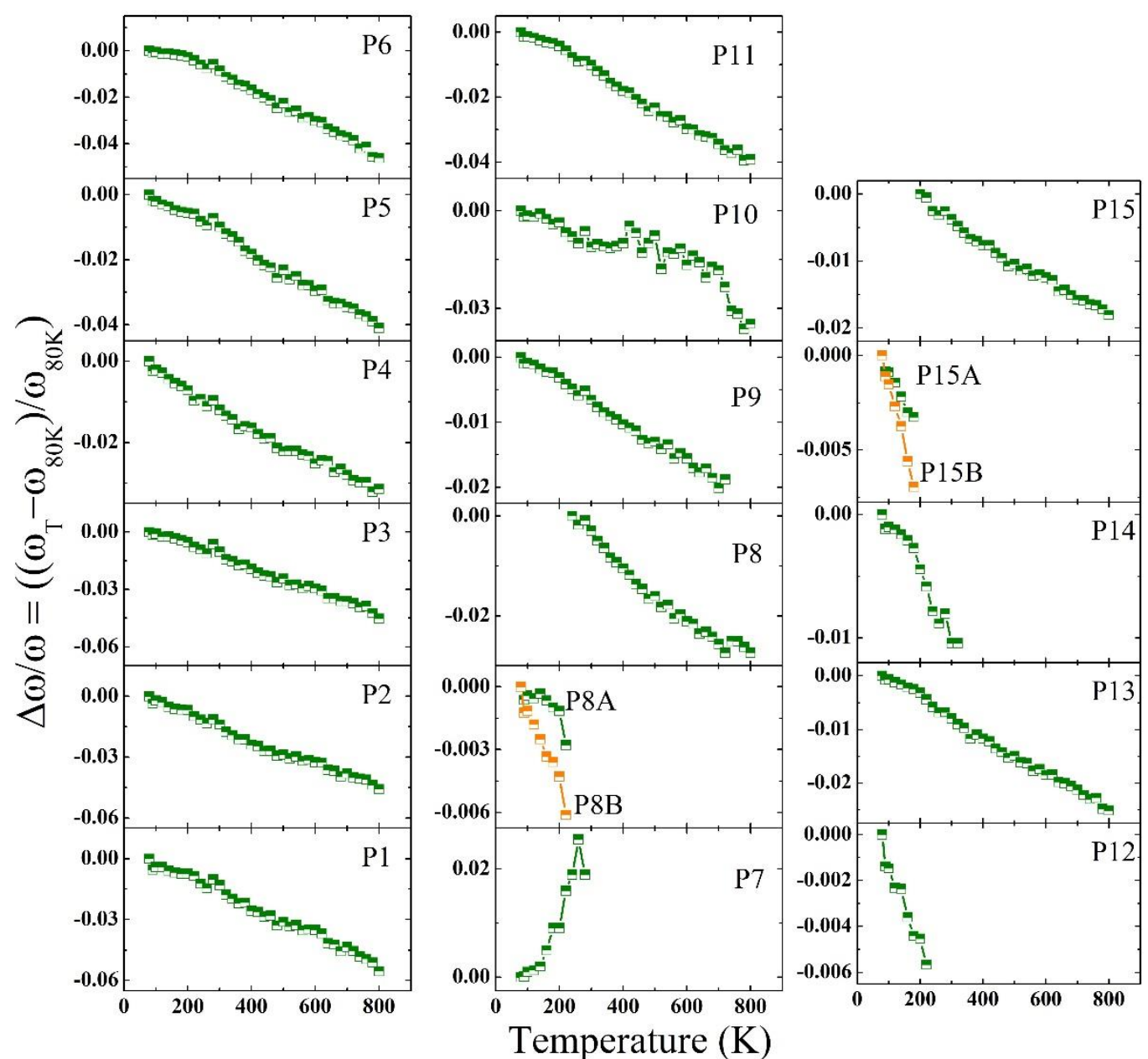

Figure S17. Plot of $\frac{\Delta \omega}{\omega} v s$ temperature. 


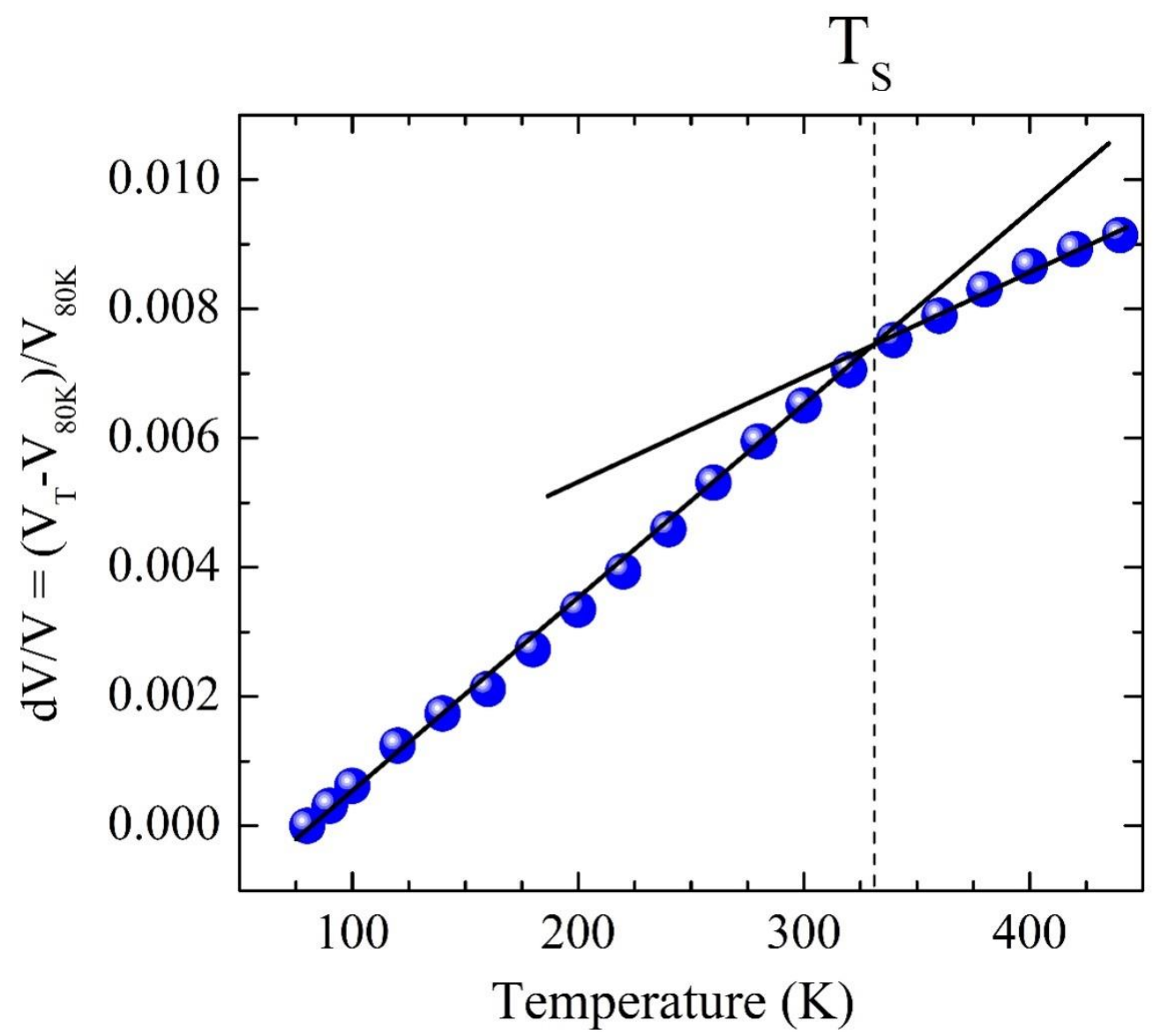

Figure S18. Plot of $\frac{\Delta V}{V}$ vs Temperature. Solid lines are guide to eye showing the deviation at $\mathrm{T}_{\mathrm{N}}$. 

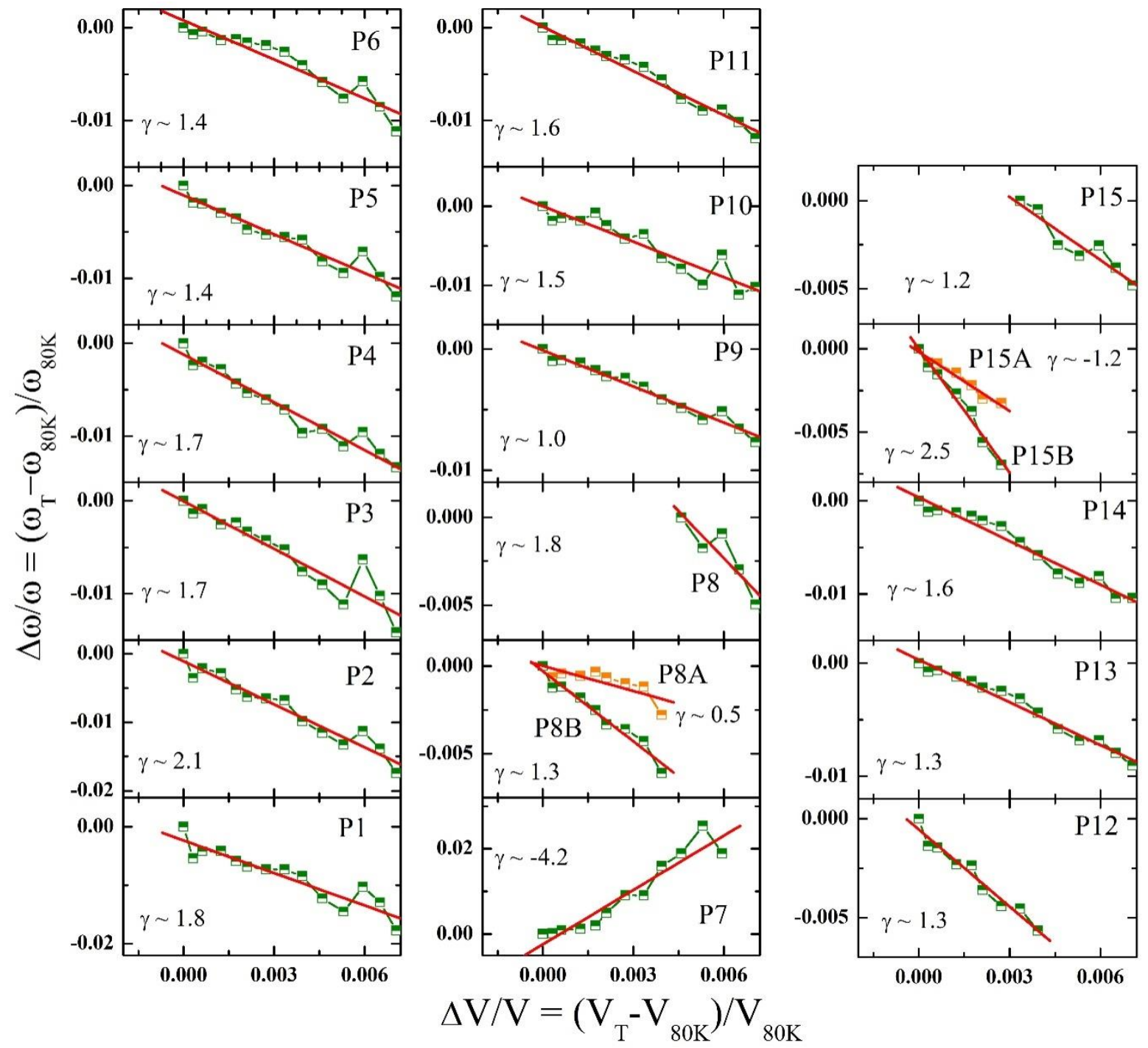

Figure S19. Plot of $\frac{\Delta \omega}{\omega}$ vs $\frac{\Delta V}{V}$. Slope of this plot gives the estimation of Grüneisen parameter $(\gamma)$. 

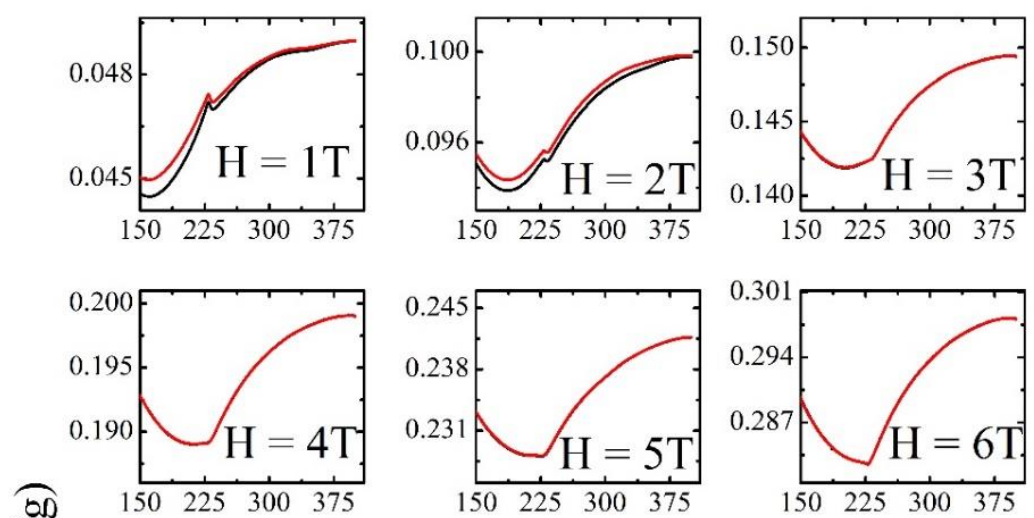

है
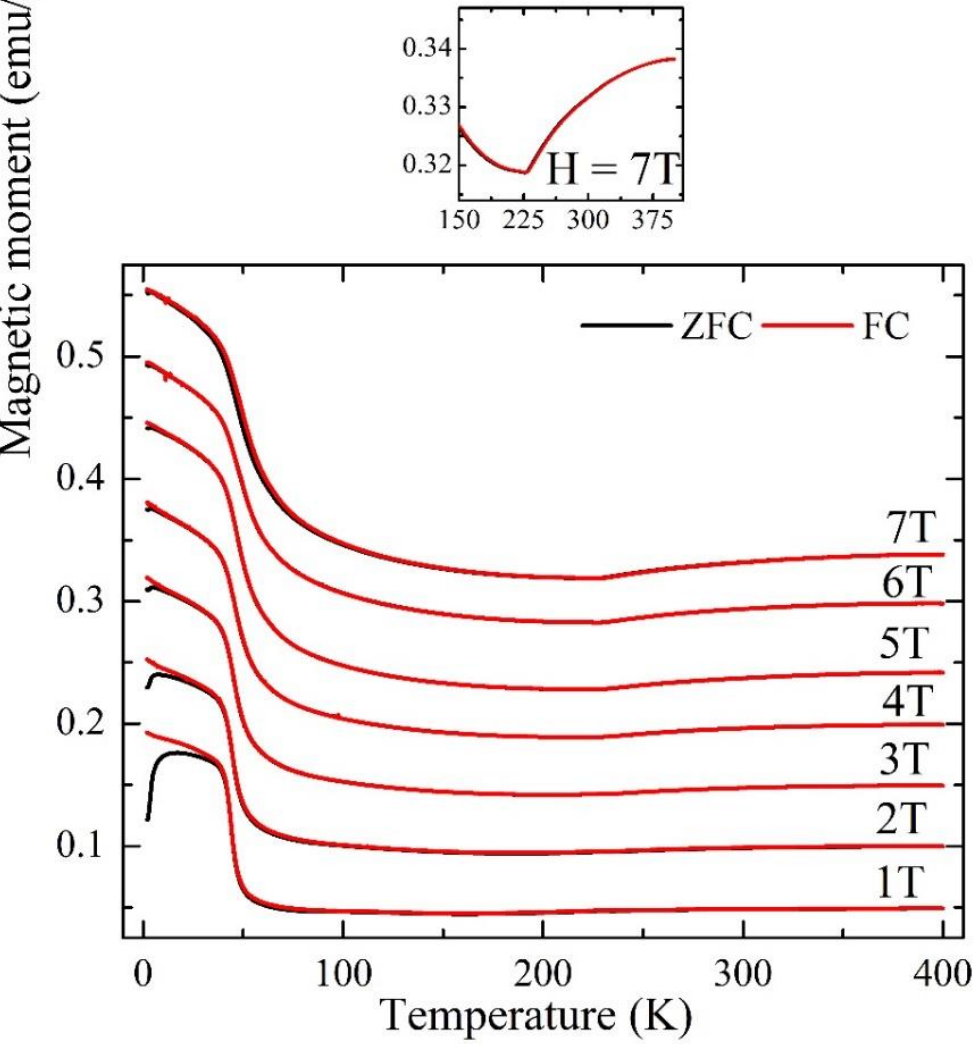

Figure S20. Temperature-dependent magnetization (ZFC and FC) measures under different applied dc magnetic fields. 


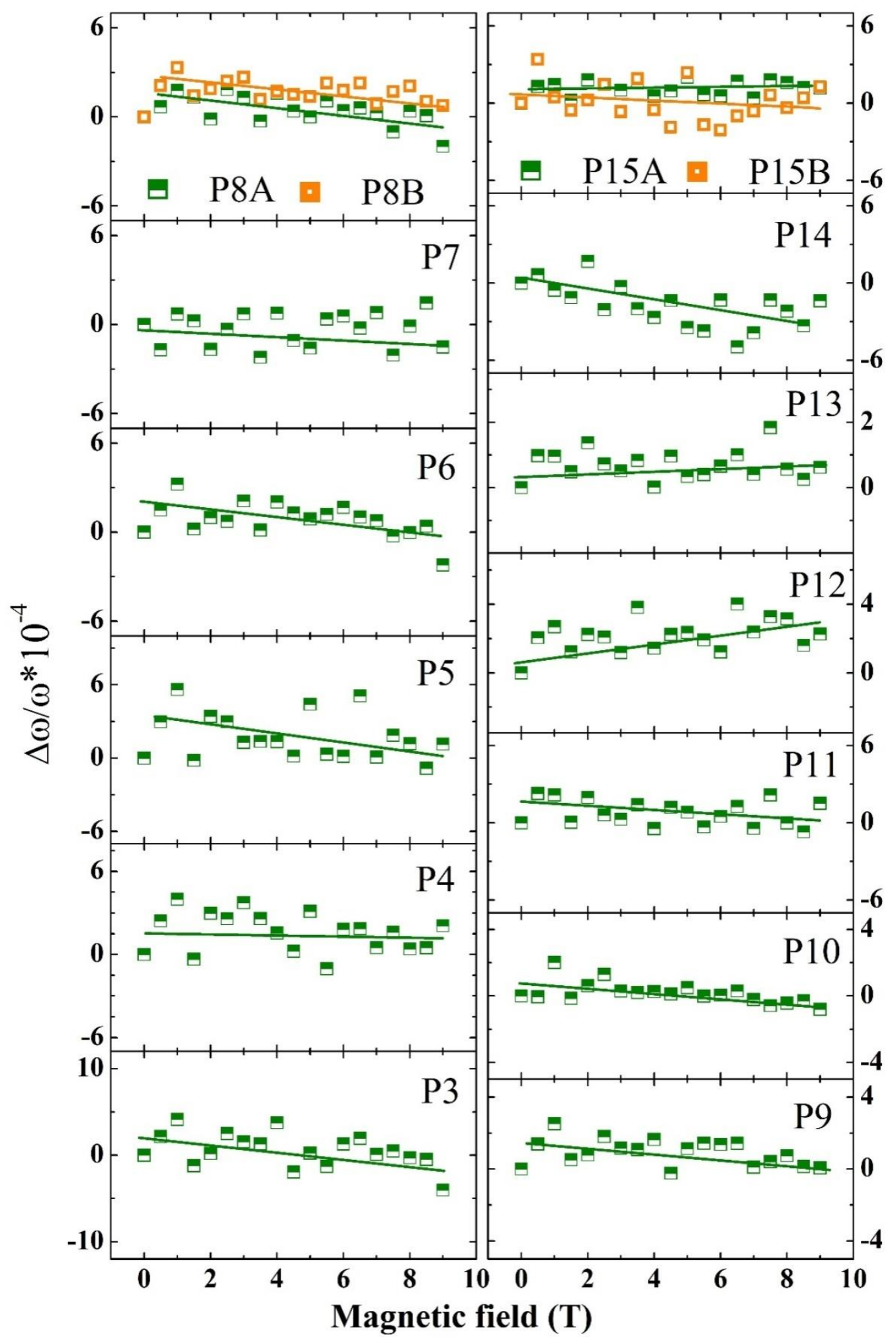

Figure S21. The plot of $\frac{\Delta \omega}{\omega}=\frac{\omega_{H}-\omega_{0 T}}{\omega_{0 T}}$ as a function of magnetic field. Solid lines are guide to eye. 
Table S5. Values for magnetostriction for a few compounds available in literature for the comparison.

\begin{tabular}{|l|l|}
\hline System & $\begin{array}{l}\text { Magnetostriction } \\
(* \mathbf{1 0}-\mathbf{6})\end{array}$ \\
\hline $\mathrm{CoMg}{ }_{x} \mathrm{Fe}_{2-\mathrm{x}} \mathrm{O} 4$ & 77 to 221 [Ref. 9] \\
\hline $\mathrm{Ce}$ & 65 [Ref. 10] \\
\hline $\mathrm{Eu}$ & $70[$ Ref. 10] \\
\hline $\mathrm{CoFe}_{2} \mathrm{O}_{4}$ & -110 [Ref. 16] \\
\hline $\mathrm{MnFe}_{2} \mathrm{O}_{4}$ & -5 [Ref. 16] \\
\hline $\mathrm{MgFe}_{2} \mathrm{O}_{4}$ & -6 [Ref. 16] \\
\hline $\mathrm{NiFe}_{2} \mathrm{O}_{4}$ & $\begin{array}{l}-24(\text { along 111) } \\
-51 \text { (along 100) (at 4.2 } \\
\text { K) [Ref. 14] }\end{array}$ \\
\hline $\mathrm{Zn}_{1-\mathrm{x}} \mathrm{Cu}_{\mathrm{x}} \mathrm{Cr}_{2} \mathrm{Se}_{4}$ & $\begin{array}{l}400 \text { to 2500 (at 100 K) } \\
\text { [Ref. 15] }\end{array}$ \\
\hline
\end{tabular}

\section{References}

1. J. J. Adkin, M.A. Hayward, $\mathrm{BaMnO}_{3-\mathrm{x}}$ revisited: a structural and magnetic study, Chem. Mater. 19 (2007) 755-762.

2. O B Korneta, T F Qi, M Ge, S Parkin, L E De Long, P Schlottmann and G Cao, "Correlated giant dielectric peaks and antiferromagnetic transitions near room temperature in pure and alkali-doped $\mathrm{BaMnO}_{3-\delta}$ ", J. Phys.: Condens. Matter 23, 435901 (2011).

3. L. Ghivelder, I. Abrego Castillo, N.McN. Alford, G.J. Tomka, P.C. Riedi, J. MacManus-Driscoll, A.K.M. Akther Hossain, L.F. Cohen, "Specific heat of La 1 ${ }_{x} \mathrm{Ca}_{x} \mathrm{MnO}_{3-\mathrm{d}}$ ", J. Mag. Mag. Mat. 189, 274 (1998).

4. B.F. Woodfield, M.L. Wilson, J.M. Byers, "Low-Temperature Specific Heat of La1${ }_{x} \mathrm{Sr}_{\mathrm{x}} \mathrm{MnO}_{3+\mathrm{d}}$ ", Phys. Rev. Lett. 78, (1997) 3201.

5. Chen Xiang-Bai, Hien Nguyen Thi Minh, YANG In-Sang, LEE Daesu, NOH TaeWon "A Raman Study of the Origin of Oxygen Defects in Hexagonal Manganite Thin Films", Chin. Phys. Lett. 29, 126103 (2012).

6. Charles Kittel, Introduction to Solid State Physics, seventh ed., Wiley, New York, 2003.

7. B. K. Tanner, "Diffraction techniques in the study of magnetic domains and magnetostriction”, Sci. Prog., Oxf. 67, 411(1981).

8. J. Kusz, S. Juszczyk and J. Warczewski, "An X-ray diffraction study of magnetostriction in $\mathrm{Zn}_{1-\mathrm{x}} \mathrm{Cu}_{\mathrm{x}} \mathrm{Cr}_{2} \mathrm{Se}_{4}(0 \cdot 2<\mathrm{x}<1 \cdot 0)$ ", J. Appl. Cryst. 21, 898(1988). 
9. P. N. Anantharamaiah and P. A. Joy, "Enhancing the strain sensitivity of $\mathrm{CoFe}_{2} \mathrm{O}_{4}$ at low magnetic fields without affecting the magnetostriction coefficient by substitution of small amounts of Mg for Fe", Phys. Chem. Chem. Phys.,18, 10516 (2016).

10. M. Doerr, M. Rotter and A. Lindbaum,"Magnetostriction in rare-earth based antiferromagnets", Advances in Physics54, 1-66 (2005).

11. S. S. Fonton and A. V. Zalesskiî,"Magnetostriction of a single crystal of hexagonal ferrite $\mathrm{BaFe}_{18} \mathrm{O}_{27}$ ”, J. Exptl. Theoret. Phys. (U.S.S.R.) 47, 1693 (1964).

12. E. Klokholm and J. Aboaf, "The saturation magnetostriction of thin polycrystallinefilms of iron, cobalt, and nickel'Journal of Applied Physics 53, 2661 (1982).

13. Abhishek Majumder, KodamUgendar, Anoop Baby K. B., VenkataraoChunchu, R. A. Mondal and G. Markandeyulu, "Anisotropy, Magnetostriction and ConverseMagnetoelectric effect in Dy substituted Ni Ferrite”, Physics Procedia 75, 238 (2015).

14. A. B. Smith and R. V. Jones, "Magnetostriction in Nickel Ferrite and Cobalt-Nickel Ferrite"Journal of Applied Physics 37, 1001 (1966).

15. J. Kusz, S. Juszczyk and J. Warczewski, "An X-ray diffraction study of magnetostriction in $\mathrm{Zn}_{1-\mathrm{x}} \mathrm{Cu}_{\mathrm{x}} \mathrm{Cr}_{2} \mathrm{Se}_{4}(0 \cdot 2<\mathrm{x}<1 \cdot 0)$ ", J. Appl. Cryst. 21, 898(1988).

16. J. Smit and H. P. J. Wijn, Ferrites, Philips Technical Library, Eindhoven, The Netherlands, 1959. 